\title{
Floquet-Liouville supermatrix approach: Time development of density-matrix operator and multiphoton resonance fluorescence spectra in intense laser fields
}

\author{
Tak-San Ho and Kwanghsi Wang \\ Department of Chemistry, University of Kansas, Lawrence, Kansas 66045 \\ Shih-I Chu* \\ Joint Institute for Laboratory Astrophysics, University of Colorado and National Bureau of Standards, \\ Boulder, Colorado 80309
}

(Received 23 September 1985)

\begin{abstract}
A Floquet-Liouville supermatrix (FLSM) approach is presented for nonperturbative treatment of the time development of the density-matrix operator of atoms and molecules exposed to intense polychromatic fields. By extending the many-mode Floquet theory (MMFT) recently developed, the time-dependent Liouville equation for the density matrix of quantum systems undergoing relaxations (due to radiative decays and collisional dampings, etc.) can be transformed into an equivalent time-independent non-Hermitian FLSM eigenvalue problem. This yields a numerically stable and computationally efficient approach for the unified treatment of nonresonant and resonant, one- and multiple-photon, steady-state and transient phenomena in nonlinear optical processes, much beyond the conventional rotating-wave-approximation (RWA) method. Connections of the FLSM approach to the MMFT in the limit of zero relaxations are also made, providing the understanding of the physical significance of FLSM supereigenvalues and eigenvectors. In addition to the exact FLSM formalism, we have also presented higher-order perturbative results, based on the extension of the generalized Van Vleck (GVV) nearly degenerate perturbation theory, appropriate for somewhat weaker fields and near-resonant processes, but beyond the RWA limit. The implementation of the GVV method in the time-independent Floquet-Liouvillian allows the reduction of the infinitedimensional FLSM into a finite-dimensional GVV-Liouville matrix, from which essential analytical results are readily obtained. As an illustration of the usefulness of the new formalism, we extend both the FLSM and the GVV methods to a formal study of the multiphoton-induced resonance fluorescence spectra of two-level systems subject to purely radiation relaxations. Both the timeaveraged power spectrum and the time-dependent physical spectrum are exploited in details, and novel new features in intense fields are pointed out.
\end{abstract}

\section{INTRODUCTION}

The investigation of nonlinear optical processes induced by intense laser fields such as multiphoton ionization and dissociation of atoms and molecules, resonance fluorescence, Raman scatterings, and wave mixings, etc., is a topic of much current interest ${ }^{1,2}$ both theoretically and experimentally. At lower fields, perturbative and diagrammatic methods are often used for nonresonant phenome$\mathrm{na},{ }^{2}$ whereas the rotating-wave approximation (RWA) is most commonly adopted for near-resonant processes. $^{3}$ The semiclassical Floquet approaches based on the Schrödinger equation, while providing nonperturbative techniques for the studies of multiphoton ionization, excitation, and dissociation processes at high fields, ${ }^{4}$ cannot be applied to processes undergoing relaxations due to radiative decays and collision dampings, etc. In this paper we present a general nonperturbative semiclassical treatment of the Liouville equation (allowing for relaxation mechanisms) for the density-matrix operator of atomic or molecular systems exposed to intense monochromatic or polychromatic fields. By extending the many-mode Floquet theory (MMFT) recently developed, ${ }^{5}$ the timedependent Liouville equation can be transformed into an equivalent time-independent Floquet-Liouville supermatrix (FLSM) eigenproblem. ${ }^{6}$ In addition to being numerically stable and computationally efficient, the FLSM method is capable of treating nonresonant and resonant, one-photon and multiphoton, steady-state and transient phenomena on an equal footing.

As an illustration of the usefulness of the FLSM method, we shall study one- and multiple-photon-induced resonance transitions and light scatterings of an ensemble of two-level atoms or molecules illuminated by a strong monochromatic field. While one-photon resonance light scatterings, fluorescence in particular, have been widely discussed, ${ }^{3,7-18}$ especially since the work of Mollow, ${ }^{9}$ the multiphoton-induced light scatterings have received little attention. ${ }^{19}$ With the advent of powerful intense lasers, we expect the experiment on these multiphoton-induced, via virtual levels, resonance effects can be accomplished in the near future. One of the major purposes of this paper is to exploit new nonlinear dynamical features in multiphoton-induced resonance fluorescence spectra.

In addition to the exact FLSM formalism, we shall present some higher-order perturbative results, appropriate for somewhat weaker fields, and near-resonant processes, but beyond the RWA limit. The most expedient 
way to accomplish this is to extend the generalized Van Vleck (GVV) nearly degenerate perturbation theory $^{5(\mathrm{~d}), 5(\mathrm{e}), 20,21}$ to the time-independent FloquetLiouvillian. This has the effect of reducing the infinitedimensional FLSM into a finite-dimensional GVVLiouville matrix, from which essential analytical results for the solutions of the density matrix as well as for the fluorescence spectrum (in both one- and multiple-photon resonance cases) can be readily obtained.

In Sec. II we present the general theory of the FLSM. This is followed by a discussion in Sec. III on the connections of the FLSM approach to the MMFT approach in the limit of zero relaxation, thus providing an understanding of the physical significance of FLSM supereigenvalues and eigenvectors. In Sec. IV the GVV nearly degenerate procedure is extended to an analytical treatment of multiphoton resonance transitions for two-level atoms or molecules in the presence of a strong laser field. In Sec. V we introduce a new scheme of the so-called time-averaged power spectrum and also derive the formula for the timedependent physical spectrum ${ }^{22-26}$ for the multiphoton resonance fluorescence by two-level systems. Results and discussions for a case study are presented in Sec. VI. This is followed by a conclusion in Sec. VII. Atomic units are used throughout unless otherwise specified.

\section{MANY-MODE FLOQUET-LIOUVILLE SUPERMATRIX APPROACH}

The time evolution of a set of $N$-level quantum systems, interacting with an $M$-mode linearly polarized polychromatic field, undergoing relaxations by Markovian processes is described by the Liouville equation ${ }^{2,27}$

$$
i \partial_{t} \hat{\rho}(t)=[\hat{H}(t), \hat{\rho}(t),]+i[\hat{R} \hat{\rho}(t)] .
$$

Here $\hat{\rho}$ is the density matrix of the system, reduced by an averaging over all irrelevant degrees of freedom action as a thermal bath, and $\hat{H}(t)=\hat{H}_{0}+\hat{V}(t) . \hat{H}_{0}$ is the unperturbed atomic Hamiltonian with eigenvalues $\left\{E_{\alpha}\right\}$ and eigenvectors $\{|\alpha\rangle\}$, namely,

$$
\hat{H}_{0}|\alpha\rangle=E_{\alpha}|\alpha\rangle, \quad \alpha=0,1,2, \ldots, N-1,
$$

and $V(t)$ is the electric dipole interaction Hamiltonian between the system and the $M$-mode classical fields,

$$
V(t)=-\sum_{i=1}^{M} \mu \cdot \varepsilon_{i} \cos \left(\omega_{i} t+\phi_{i}\right),
$$

where $\mu$ is the atomic dipole moment, and $\varepsilon_{i}, \omega_{i}$, and $\phi_{i}$ are, respectively, the field amplitude, frequency, and phase of the $i$ th field. The relaxation term $[\hat{R} \hat{\rho}(t)]$ consists of $T_{1}$ (population damping) and $T_{2}$ (coherent damping) mechanisms which are due to the coupling of the atomic system to the thermal bath by radiative decays and collisions, etc. More explicitly, it can be written $\operatorname{as}^{27}$

$$
\begin{aligned}
& (\hat{R} \hat{\rho})_{\alpha \alpha}=-\Gamma_{\alpha \alpha} \rho_{\alpha \alpha}+\sum_{\beta(\neq \alpha)} \gamma_{\beta \alpha} \rho_{\beta \beta}\left(T_{1} \text { process }\right), \\
& (\hat{R} \hat{\rho})_{\alpha \beta}=-\Gamma_{\alpha \beta} \rho_{\alpha \beta}, \quad \alpha \neq \beta\left(T_{2} \text { process }\right) .
\end{aligned}
$$

The relaxation constants appearing in Eqs. (4) and (5) are connected by the relations

$$
\Gamma_{\alpha \beta}=\frac{1}{2}\left(\Gamma_{\alpha \alpha}+\Gamma_{\beta \beta}\right)+\Gamma_{\alpha \beta}^{\prime}
$$

and

$$
\Gamma_{\alpha \beta}=\Gamma_{\beta \alpha},
$$

with the factor $\Gamma_{\alpha \beta}^{\prime}$ usually called pure dephasing. The feeding term $\gamma_{\alpha \beta}$ is generally not symmetrical, i.e., $\gamma_{\alpha \beta} \neq \gamma_{\beta \alpha}$, but can be related to $\Gamma_{\alpha \alpha}$ in the case of closed systems, namely,

$$
\Gamma_{\alpha \alpha}=\sum_{\beta} \gamma_{\alpha \beta}
$$

In the following, we shall only consider the case of closed systems in the development of our approach, although it is straightforward to extend it to the case of open systems.

To facilitate the evaluation of the reduced densitymatrix operator $\hat{\rho}(t)$ in Eq. (1), we reformulate the equation in an operator (a tetradic, or Liouville) space ${ }^{8}$ spanned by the basis $\{|\alpha \beta\rangle \equiv|\alpha\rangle\langle\beta| ; \alpha$ and $\beta=0$, $1, \ldots, N-1\}$ as an inhomogeneous superoperator equation, namely,

$$
i \partial_{t} \rho(t)=\hat{L}(t) \rho(t)+i \mathbf{f} .
$$

Here $\rho(t)$ is the supervector defined by

$$
\rho(t)=\sum_{\alpha, \beta} \rho_{\alpha \beta}|\alpha\rangle\langle\beta| ;
$$

$\hat{L}(t)$, the superoperator or Liouvillian, in matrix form, is given by

$$
\begin{aligned}
\hat{L}_{00 ; \mu \nu}(t) \equiv & \langle 00|\hat{L}(t)| \mu v\rangle \\
= & \hat{H}_{0 \mu}(t) \delta_{0 v}-\hat{H}_{\nu 0}(t) \delta_{0 \mu} \\
& \quad-i\left(\left(\Gamma_{00}+\gamma_{0}\right) \delta_{\mu 0} \delta_{\nu 0}\right. \\
& \left.\quad+\sum_{\beta \neq 0}\left(1-\delta_{\beta \mu}\right) \gamma_{\beta 0} \delta_{\mu v}\left(1-\delta_{\mu 0}\right)\right],
\end{aligned}
$$

$$
\begin{aligned}
\hat{L}_{\alpha \beta ; \mu \nu}(t) \equiv & \langle\alpha \beta|\hat{L}(t)| \mu v\rangle \\
= & \hat{H}_{\alpha \mu}(t) \delta_{\beta v}-\hat{H}_{\nu \beta}(t) \delta_{\alpha \mu} \\
& -i\left(\Gamma_{\mu \nu} \delta_{\alpha \mu} \delta_{\beta v}-\gamma_{\mu \beta} \delta_{\alpha \beta} \delta_{\mu v}\right) \\
& (\alpha \neq 0 \text { and /or } \beta \neq 0) ;
\end{aligned}
$$

and finally, $f$ is the source supervector, defined by

$$
\mathbf{f}_{\mu \nu}=\gamma_{0} \delta_{\mu 0} \delta_{v 0}
$$

where $\gamma_{0} \equiv \sum_{\beta \neq 0} \gamma_{\beta 0}$, assuming $|0\rangle$ is the ground level. Note that in deriving Eqs. (9) and (11)-(13), use has been made of the relationship $\operatorname{Tr} \hat{\rho}(t)=1$ (appropriate for closed systems) and the following basic properties of the Liouville space: (i) inner product,

$$
\langle\alpha \beta \mid \mu \nu\rangle \equiv \operatorname{Tr}\{|\beta\rangle\langle\alpha \mid \mu\rangle\langle\nu|\},
$$

and (ii) matrix elements of superoperator $\hat{O}$,

$$
\langle\alpha \beta|\hat{O}| \mu \nu\rangle \equiv \operatorname{Tr}\{|\beta\rangle\langle\alpha|\hat{O}| \mu\rangle\langle\nu|\} .
$$


The superoperator $\hat{L}(t)$ defined by Eqs. (11) and (12) is nonsingular, which possesses distinct advantages as will be shown below.

The inhomogeneous equation (9) now can be solved by using the method developed to solve the Schrödinger equation. By invoking the MMFT, ${ }^{4,5}$ we can transform the time-dependent superoperator equation (9) into an equivalent time-independent infinite-dimensional supereigenvalue problem, ${ }^{28}$ namely,

$$
\begin{array}{r}
\sum_{\sigma, \tau\{k\}}\left\langle\alpha \beta ;\{m\}\left|\hat{L}_{F}\right| \sigma \tau ;\{k\}\right\rangle\left\langle\sigma \tau ;\{k\} \mid \Omega_{\mu v,\{n\}}\right\rangle \\
=\Omega_{\mu v:\{n\}}\left\langle\alpha \beta ;\{m\} \mid \Omega_{\mu v ;\{n\}}\right\rangle,
\end{array}
$$

where $\hat{L}_{F}$ is the time-independent many-mode FloquetLiouvillian defined in terms of the generalized tetradicFourier basis $|\alpha \beta ;\{m\}\rangle \equiv|\alpha\rangle\langle\beta|\otimes|\{m\}\rangle$, with $\{m\}$ $=m_{1}, m_{2}, \ldots, m_{M}$. More explicitly, $\hat{L}_{F}$ can be written, in matrix form, as

$$
\begin{aligned}
&\left\langle\alpha \beta ;\{m\}\left|\hat{L}_{F}\right| \mu v ;\{n\}\right\rangle \\
&=\hat{L}_{\alpha \beta ; \mu \nu}^{\{m-n\}}+\sum_{i=1}^{M} m_{i} \omega_{i} \delta_{\alpha \mu} \delta_{\beta \nu} \delta_{\{m\} ;\{n\}},
\end{aligned}
$$

with

$$
\begin{aligned}
& \hat{L}_{\alpha \beta ; \mu \nu}^{\{m\}}=\left\{\begin{array}{l}
\hat{H}_{{ }_{\mu}}^{\{m\}} \delta_{0 \nu}-\hat{H}_{\nu}^{\{m\}} \delta_{0 \mu}-i\left[\left(\Gamma_{00}+\gamma_{0}\right) \delta_{0 \mu} \delta_{0 v}+\sum_{\beta \neq 0}\left(1-\delta_{\beta \mu}\right) \gamma_{\beta 0} \delta_{\mu \nu}\left(1-\delta_{\mu 0}\right)\right] \delta_{\{m\} ;\{0\}} \quad \text { if } \alpha=0 \text { and } \beta=0 \\
\hat{H}_{\alpha \mu}^{\{m\}} \delta_{\beta \nu}-\hat{H}_{\nu \beta}^{\{m\}} \delta_{\alpha \mu}-i\left(\Gamma_{\mu \nu} \delta_{\mu \alpha} \delta_{\beta \nu}-\gamma_{\mu \beta} \delta_{\alpha \beta} \delta_{\mu \nu}\right) \delta_{\{m\} ;\{0\}} \text { otherwise, }
\end{array}\right. \\
& \hat{H}_{\alpha \beta}^{\{m\}}=E_{\alpha} \delta_{\alpha \beta} \delta_{\{m\} ;\{0\}}+\sum_{i=1}^{M} \hat{V}_{\alpha \beta}^{(i)}\left(\delta_{m_{i}, 1} e^{i \phi_{i}}+\delta_{m_{i},-1} e^{-i \phi_{i}}\right) \delta_{\{m\} ;\{0\}}^{(i)}, \\
& \hat{V}_{\alpha \beta}^{(i)}=-\frac{1}{2}\left\langle\alpha\left|\mu \cdot \varepsilon_{i}\right| \beta\right\rangle, \\
& \delta_{\{m\} ;\{n\}}=\prod_{i=1}^{M} \delta_{m_{i}, n_{i}},
\end{aligned}
$$

and

$$
\delta_{\{m\} ;\{n\}}^{(i)}=\prod_{\substack{j=1 \\ j \neq i}}^{M} \delta_{m_{j}, n_{j}},
$$

where $\delta_{m, n}$ is the Kronecker delta function. The structure of the Floquet-Liouville supermatrix $\hat{L}_{F}$, which is nonHermitian, is illustrated in Fig. 1 for the two-level twomode case. The multiply periodic structure of $\hat{L}_{F}$ renders the following important periodic relationships for its supereigenvalues $\Omega_{\mu v ;\{n\}}$ and supereigenvectors $\left|\Omega_{\mu v,\{n\}}\right\rangle$ :

$$
\Omega_{\mu v,\{n+k\}}=\Omega_{\mu v,\{n\}}+\sum_{i=1}^{M} k_{i} \omega_{i}
$$

and

$\left\langle\alpha \beta ;\{m+k\} \mid \Omega_{\mu v,\{n+k\}}\right\rangle=\left\langle\alpha \beta ;\{m\} \mid \Omega_{\mu v,\{n\}}\right\rangle$.

Owing to the non-Hermitian nature of $\hat{L}_{F}$, there exists biorthogonal relationships,

$$
\left\langle\Omega_{\sigma \tau,\{k\}}^{\prime} \mid \Omega_{\mu v,\{n\}}\right\rangle=\delta_{\sigma \mu} \delta_{\tau \nu} \delta_{\{k\} ;\{n\}},
$$

where $\left|\Omega_{\sigma \tau,\{k\}}^{\prime}\right\rangle$ are eigenvectors of $\hat{L}_{F}^{\dagger}$, namely,

$$
\hat{L}_{F}^{\dagger}\left|\Omega_{\sigma \tau ;\{k\}}^{\prime}\right\rangle=\Omega_{\sigma \tau ;\{k\}}^{\prime}\left|\Omega_{\sigma \tau ;\{k\}}^{\prime}\right\rangle
$$

and

$$
\Omega_{\sigma \tau,\{k\}}^{\prime}=\Omega_{\sigma \tau,\{k\}}^{*} .
$$

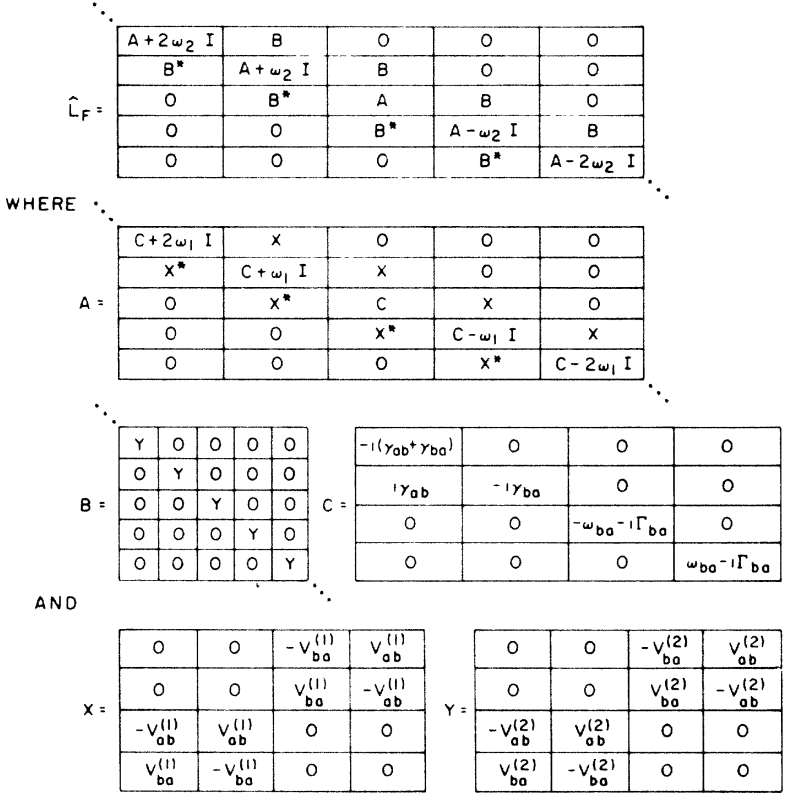

FIG. 1. Structure of the Floquet-Liouville supermatrix $\hat{L}_{F}$ for the case of two-level systems (with level spacing $\omega_{b a}$ ) in linearly polarized bichromatic fields. $\omega_{1}$ and $\omega_{2}$ are the two radiation frequencies, $V_{a b}^{(i)}(i=1,2)$ are the electric dipole couplings, and $\gamma_{a b}, \gamma_{b a}$, and $\Gamma_{b a}=\left(\gamma_{a b}+\gamma_{b a}\right) / 2$ are relaxation parameters. 
Furthermore, the negativity of the imaginary part of all of the diagonal elements of $\hat{L}_{F}$ guarantees that

$$
\operatorname{Im}\left(\Omega_{\mu v,\{n\}}\right)<0 .
$$

In terms of the supereigenvalues and eigenvectors of the
Floquet-Liouvillian $\hat{L}_{F}$, the reduced density supervector $\rho(t)$ of Eq. (9) can be expressed as

$$
\rho(t)=\hat{U}\left(t ; t_{0}\right) \rho\left(t_{0}\right),
$$

where $\hat{U}$ is the non-Hermitian superevolution operator given by, in matrix form,

$$
\begin{aligned}
\hat{U}_{\alpha \beta ; \mu \nu}\left(t ; t_{0}\right)=\sum_{\{m\}} \mid\left\langle\alpha \beta ;\{m\}\left|\exp \left[-i \hat{L}_{F}\left(t-t_{0}\right)\right]\right| \mu \nu ;\{0\}\right\rangle \\
+\gamma_{0} \delta_{\mu \nu} \sum_{\sigma, \tau\{k\}}\left\langle\alpha \beta ;\{m\} \mid \Omega_{\sigma \tau,\{k\}}\right\rangle\left\langle\Omega_{\sigma \tau,\{k\}}^{*} \mid 00 ;\{0\}\right\rangle \\
\left.\quad \times\left\{1-\exp \left[-i \Omega_{\sigma \tau,\{k\}}\left(t-t_{0}\right)\right]\right\} / i \Omega_{\sigma \tau,\{k\}}\right) \exp \left(i \sum_{j=1}^{M} m_{j} \omega_{j} t\right),
\end{aligned}
$$

and $\rho\left(t_{0}\right)$ denotes the initial state of the system at time $t_{0}$. Furthermore, since $\operatorname{Im} \Omega<0$ for all $\Omega$, the reduced density matrix has a simple asymptotic form at large times $t \rightarrow \infty$,

$$
\rho_{\alpha \beta}(t) \rightarrow \gamma_{0} \sum_{\{m\}}\left(\sum_{\sigma, \tau\{k\}}\left\langle\alpha \beta ;\{m\} \mid \Omega_{\sigma \tau ;\{k\}}\right\rangle\left\langle\Omega_{\sigma \tau,\{k\}}^{*} \mid 00,\{0\}\right\rangle / i \Omega_{\sigma \tau,\{k\}}\right) \exp \left(i \sum_{j=1}^{M} m_{j} \omega_{j} t\right) \text { as } t \rightarrow \infty,
$$

which is independent of the initial state $\rho\left(t_{0}\right)$ and is oscillatory in the course of the time, dictated by the Fourier terms $\exp \left(i \sum_{j=1}^{M} m_{j} \omega_{j} t\right)$, rather than completely stationary, as would be the case in the RWA limit. For the single-mode case, i.e., $M=1, \rho_{\alpha \beta}(t)$ as $t \rightarrow \infty$ behaves periodically in time with the period $2 \pi / \omega, \omega$ being the single-mode laser frequency. For an ensemble of $N$-level systems, we allow for the random interaction durations for each member of the systems with the laser fields and, therefore, can average the quantity $\rho_{\alpha \beta}(t)$ over a sufficiently long period $T \gg \max \left\{2 \pi / \omega_{i}\right\}, i=1,2, \ldots, M$. This average results in the long time-averaged reduced density-matrix elements

$$
\begin{gathered}
\bar{\rho}_{\alpha \beta}=\gamma_{0} \sum_{\sigma, \tau} \sum_{k}\left(i \Omega_{\sigma \tau,\{k\}}\right)^{-1}\left\langle\alpha \beta ;\{0\} \mid \Omega_{\sigma \tau,\{k\}}\right\rangle \\
\times\left\langle\Omega_{\sigma \tau,\{k\}}^{*} \mid 00 ;\{0\}\right\rangle .
\end{gathered}
$$

We note that because of the parity considerations, via the electric dipole approximation, the unperturbed tetradicFloquet states $|\alpha \alpha ;\{m\}\rangle$ corresponding to the diagonal density-matrix elements $\rho_{\alpha \alpha}(t)$ are only coupled to states $|\beta \beta ;\{n\}\rangle$ with $\sum_{i=1}^{M}\left\{\left|n_{i}\right|+\left|m_{i}\right|\right\}$ even integers and to states $|\mu v ;\{k\}\rangle$ with $\mu \neq v$ and $\sum_{i=1}^{M}\left\{\left|k_{i}\right|+\left|m_{i}\right|\right\}$ odd integers. This selection rule implies that the long time-averaged coherences, i.e., $\bar{\rho}_{\alpha \beta}, \alpha \neq \beta$, are always zero in the observational (laboratory) frame, while the long time-averaged population $\bar{\rho}_{\alpha \alpha}$ 's are, in general, nonvanishing. The time-dependent coherence $\rho_{\alpha \beta}(t), \alpha \neq \beta$, is frame dependent.

\section{CONNECTION TO THE MANY-MODE FLOQUET HAMILTONIAN APPROACH}

To identify the physical meanings of the supereigenvalue $\Omega$ 's and supereigenvectors $|\Omega\rangle$ 's of the $\hat{L}_{F}$ in Sec. II, we shall now consider the case where all the relaxa- tions are quenched. Here, the density-matrix operator $\hat{\rho}(t)$ can be formed by the state wave function $|\Psi(t)\rangle$ of the system, namely,

$$
\hat{\rho}(t)=|\Psi(t)\rangle\langle\Psi(t)|,
$$

where the state wave function $|\Psi(t)\rangle$ is governed by the time-dependent Schrödinger equation

$$
i \partial_{t}|\Psi(t)\rangle=\hat{H}(t)|\Psi(t)\rangle,
$$

and the total Hamiltonian $\hat{H}(t)$ has been defined in the beginning of Sec. II. By invoking the many-mode Floquet theory, we can transform Eq. (34) into an eqivalent timeindependent eigenvalue equation ${ }^{4,5}$

$$
\begin{aligned}
\sum_{\beta} \sum_{\{k\}}\left\langle\alpha ;\{m\}\left|\hat{H}_{F}\right| \beta ;\{k\}\right\rangle\left\langle\beta ;\{k\} \mid \lambda_{\mu ;\{n\}}\right\rangle \\
=\lambda_{\mu ;\{n\}}\left\langle\alpha ;\{m\} \mid \lambda_{\mu ;\{n\}}\right\rangle,
\end{aligned}
$$

where $\hat{H}_{F}$ is the time-independent Hermitian Floquet Hamiltonian. In terms of the generalized Floquet basis $|\alpha ;\{m\}\rangle \equiv|\alpha\rangle \otimes|\{m\}\rangle, \hat{H}_{F}$ can be expressed as

$$
\begin{aligned}
\left\langle\alpha ;\{m\}\left|\hat{H}_{F}\right| \beta ;\{k\}\right\rangle & \\
& =\hat{H}_{\alpha \beta}^{\{m-k\}}+\sum_{i=1}^{M} m_{i} \omega_{i} \delta_{\alpha \beta} \delta_{\{m\},\{k\}},
\end{aligned}
$$

where $\hat{H}_{\alpha \beta}^{\{n\}}$ has been defined in Eq. (19). The (real) quasienergies $\lambda_{\mu ;\{n\}}$ and quasienergy eigenvectors $\left|\lambda_{\mu ;\{n\}}\right\rangle$ possess the following periodic properties:

$$
\lambda_{\mu ;\{n+k\}}=\lambda_{\mu ;\{n\}}+\sum_{i=1}^{M} k_{i} \omega_{i}
$$

and

$$
\left\langle\alpha ;\{m+k\} \mid \lambda_{\mu ;\{n+k\}}\right\rangle=\left\langle\alpha ;\{m\} \mid \lambda_{\mu ;\{n\}}\right\rangle .
$$

By directly comparing Eq. (16) with (35) and (17) with (36) and making use of relations (18), (19), (23), (24), (37), 
and (38), we arrive at the following relations:

$$
\begin{aligned}
\left\langle\alpha \beta ;\{m\}\left|\hat{L}_{F}^{(0)}\right| \mu \nu ;\{n\}\right\rangle & =\left\langle\alpha ;\{m\}\left|\hat{H}_{F}\right| \mu ;\{n\}\right\rangle \delta_{\nu \beta} \\
& -\left\langle v ;\{m\}\left|\hat{H}_{F}\right| \beta ;\{n\}\right\rangle \delta_{\alpha \mu} \\
& +\sum_{i=1}^{M} m_{i} \omega_{i} \delta_{\alpha \mu} \delta_{v \beta} \delta_{\{m\} ;\{n\}}, \\
\Omega_{\mu v ;\{n\}}^{(0)} & =\lambda_{\mu ;\{0\}}-\lambda_{v ;\{0\}}+\sum_{i=1}^{M} n_{i} \omega_{i},
\end{aligned}
$$

and

$$
\begin{aligned}
& \left\langle\alpha \beta ;\{m\} \mid \Omega_{\mu v,\{0\}}^{(0)}\right\rangle \\
& =\sum_{\{n\}}\left\langle\alpha ;\{n\} \mid \lambda_{\mu ;\{0\}}\right\rangle\left\langle\lambda_{v,\{0\}} \mid \beta ;\{n-m\}\right\rangle,
\end{aligned}
$$

where the superscript $(0)$ appearing in the superoperator $\hat{L} \stackrel{(0)}{F}$, supereigenvalues $\Omega^{(0)}$, and supereigenvectors $\left|\Omega^{(0)}\right\rangle$ indicates that all relaxations in Eqs. (4) and (5) have been set to zero. Equation (40) suggests that (i) there exists $N$ zero supereigenvalues $\Omega_{\mu \mu ;\{0\}}^{(0)}=0, \mu=0,1,2, \ldots, N-1$, for the Floquet Liouvillian $\hat{L}_{F}^{(0)}$ defined in Eq. (39), and (ii) the supereigenvalues $\Omega_{\mu v,\{0\}}^{(0)}$ for $\mu \neq v$ are the differences between two quasienergies $\lambda_{\mu ;\{0\}}$ and $\lambda_{\nu,\{0\}}$ and thus characterize the "difference spectrum" of the multiphoton process. Equation (41) shows how the supereigenvectors $\left|\Omega_{\mu v,\{0\}}^{(0)}\right\rangle$ are related to the dissipationless quasienergy eigenvectors $\left|\lambda_{\mu ;\{0\}}\right\rangle$ and $\left|\lambda_{v,\{0\}}\right\rangle$.

Other quantities of interest are the long time-averaged density-matrix elements in the limit of no relaxations. Assuming the initial condition $\rho_{\alpha \beta}\left(t_{0}\right)=\delta_{\alpha 0} \delta_{\beta 0}$, and averaging $\hat{\rho}(t) \equiv \hat{\rho}\left(t-t_{0} ; t_{0}\right)$ over first the random initial times $t_{0}$ and then all possible elapsed times $t-t_{0}$, we obtain

$\bar{\rho}_{\alpha \beta}=\sum_{\mu=0}^{N-1}\left\langle\alpha \beta ;\{0\} \mid \Omega_{\mu \mu ;\{0\}}^{(0)}\right\rangle\left\langle\Omega_{\mu \mu ;\{0\}}^{(0)} \mid 00 ;\{0\}\right\rangle$,

which is to be compared with Eq. (32) where relaxation terms are nonvanishing. Furthermore, making use of relations Eqs. (40) and (41), the long time-averaged population at level $\beta$ becomes

$\bar{\rho}_{B \beta}=\sum_{\mu=0}^{N-1} \sum_{\{n\}} \sum_{\{m\}}\left|\left\langle\beta ;\{m\} \mid \lambda_{\mu ;\{n\}}\right\rangle \cdot\left\langle\lambda_{\mu ;\{n\}} \mid 0 ;\{0\}\right\rangle\right|^{2}$,

$$
\hat{L}_{\mathrm{GVV}}=\left[\begin{array}{ccc}
-i\left(\gamma_{a b}+\gamma_{b a}\right) & 0 & \frac{1}{2} u \\
i \gamma_{a b} & -i \gamma_{b a} & -\frac{1}{2} u \\
\frac{1}{2} u & -\frac{1}{2} u & -(\Delta+\delta)-i \Gamma_{b a} \\
-\frac{1}{2} u & \frac{1}{2} u & 0
\end{array}\right.
$$

where $\gamma_{a b}$ and $\gamma_{b a}$ are feeding rates and $\Gamma_{b a}$ is the coherence damping rate discussed previously due to spontaneous emission, collisions, etc. In the pure radiative case, $\gamma_{a b}=0$ and $\Gamma_{b a}=\frac{1}{2} \gamma_{b a}$, with $\gamma_{b a}$ the spontaneous decay which is simply what we have derived previously using the Floquet Hamiltonian method based on the Schrödinger equation. ${ }^{4,5,29}$

\section{GENERALIZED VAN VLECK NEARLY DEGENERATE PERTURBATIVE TREATMENT OF THE FLOQUET-LIOUVILLIAN}

The general theory outlined in Secs. II and III enables us to explore nonlinear optical processes in intense polychromatic fields much beyond the limit of the oftenused RWA. To exploit the analytical properties of multiphoton processes undergoing relaxations, we shall now extend the generalized Van Vleck nearly degenerate perturbation theory ${ }^{5(d), 5(e), 20,21}$ to the Floquet-Liouvillian. This reduces the infinite-dimensional Floquet-Liouville supermatrix $\hat{L}_{F}$ into an effective non-Hermitian finitedimensional GVV-Liouvillian $\hat{L}_{\mathrm{GVV}}$, from which essential multiphoton dynamics can be readily obtained either analytically or numerically.

In a proper rotating frame ${ }^{5(\mathrm{~d})}$ (not the RWA) [see Eq. (51) below for an example], the density-matrix operator $\rho(t)$ satisfies approximately the GVV-Liouville equation, namely,

$$
i \partial_{t} \rho(t)=\hat{L}_{\mathrm{GVv}} \rho(t)+i \mathbf{f}_{\mathrm{GVV}}
$$

where $f_{G V V}$ is the GVV-source supervector. Without loss of generality, we shall consider the specific case of a system of dipole-allowed two-level atoms undergoing $(2 n+1)$-photon resonance transition in the presence of a single monochromatic linearly polarized laser field characterized by the frequency $\omega_{L}$ and amplitude $\varepsilon_{L}$. The two-level $|a\rangle$ and $|b\rangle\left(E_{a}<E_{b}\right)$ are of opposite parity and the phase of the laser field is assumed zero, i.e., $\phi_{L}=0$.

When the resonance condition $\omega_{b a} \equiv E_{b}-E_{a}$ $\cong(2 n+1) \omega_{L}$, with positive integers $n$, is satisfied, the unperturbed tetradic-Floquet states $|a a ; m\rangle,|b b ; m\rangle$, $|a b ; m+(2 n+1)\rangle$, and $|b a ; m-(2 n+1)\rangle$, with arbitrary integer $m$, form a four-dimensional nearly degenerate set and span the GVV "model space." In terms of this model space, the effective GVV-Liouvillian $\hat{L}_{\mathrm{GVV}}$ has the following matrix form:

$$
\left.\begin{array}{c}
-\frac{1}{2} u \\
\frac{1}{2} u \\
0 \\
\Delta+\delta)-i \Gamma_{b a}
\end{array}\right]
$$

rate from the upper level $|b\rangle$ to the ground level $|a\rangle$. The detuning $\Delta$ is defined as

$$
\Delta \equiv \omega_{b a}-(2 n+1) \omega_{L},
$$


and the resonance shift $\delta$, corrected to second order in $b$ ( $\equiv-\frac{1}{2}\left\langle a\left|\mu \cdot \varepsilon_{L}\right| b\right\rangle$ ), and the effective Rabi frequency $u$, corrected to $(2 n+1)$ th order (in $b$ ), are given, respectively, by

$$
\delta= \begin{cases}\frac{2 b^{2}}{\omega_{b a}+\omega_{L}}, & n=0 \\ \frac{4 b^{2} \omega_{b a}}{\omega_{b a}^{2}-\omega_{L}^{2}}, & n \neq 0\end{cases}
$$

and

$$
\frac{1}{2} u=(-1)^{n}\left(\frac{b}{2 \omega_{L}}\right)^{2 n} \frac{b}{(n !)^{2}} \text { for all } n .
$$

We should mention that the quantity $u$ can be expressed more accurately for each $(2 n+1)$-photon resonance process at the expense of a general form such as Eq. (48), e.g.,

$$
\frac{1}{2} u=b-\frac{b^{3}}{\left(\omega_{b a}+\omega_{L}\right)^{2}}, n=0
$$

and

$$
\frac{1}{2} u=-\frac{4 b^{3} \omega_{L}}{\left(\omega_{b a}-\omega_{L}\right)^{2}\left(\omega_{b a}+\omega_{L}\right)}, \quad n=1,
$$

etc.

There are several important points here concerning the GVV-Liouvillian $\hat{L}_{\mathrm{GVV}}$ of Eq. (45). First, in the case of one-photon resonance transition, i.e., $n=0$, the GVVLiouvillian $\hat{L}_{\mathrm{GVv}}$ reduces to the commonly used RWALiouvillian when neglecting the resonance shift $\delta$ and terms higher than first order in $b$ in the effective Rabi frequency $u$, i.e., $\frac{1}{2} u=b$. Second, in the case of $(2 n+1)$-photon resonance transition with $n \neq 0$, the GVV-Liouvillian $\hat{L}_{\mathrm{GVv}}$ resembles a single quasiparticle process carried by an effective coupling term $u$, which, of course, has a leading term in the $(2 n+1)$ th order in $b$. Finally, in the one-photon case, the shift $\delta$, of the second order in $b$, is one order of magnitude smaller than the Rabi frequency $b$, whereas in the $(2 n+1)$-photon case, $n \geq 1$, the resonance shift $\delta$, always of the second order in $b$, is orders of magnitude greater than the corresponding effective Rabi frequency $u$, which is of the $(2 n+1)$ th order in $b$. This last point plays an important role in achieving the saturation of the population distribution among various levels through multiphoton resonance transition, or pumping, which, in turn, determines the intensity of the lights scattered by the atoms.

In the rotating frame defined by the transformation

$$
\hat{R}(t) \equiv\left[\begin{array}{cc}
1 & 0 \\
0 & e^{i(2 n+1) \omega_{L} t}
\end{array}\right),
$$

the density-matrix operator $\rho(t)$ obeys Eq. (44) with the GVV-Liouvillian $\hat{L}_{\mathrm{GVV}}$ defined by Eq. (45), and the source supervector $\mathbf{f}_{\mathrm{GVV}}$ becomes

$$
\mathbf{f}_{\mathrm{GVV}}=\left(\begin{array}{c}
\gamma_{b a} \\
0 \\
0 \\
0
\end{array}\right)
$$

in the two-level one-mode case. The GVV-Liouville equation can be solved most expediently by first making the Laplace transform ${ }^{30}$ of Eq. (44), yielding an algebraic equation

$$
\left[\begin{array}{cccc}
s+\left(\gamma_{a b}+\gamma_{b a}\right) & 0 & \frac{i}{2} u & -\frac{i}{2} u \\
-\gamma_{a b} & s+\gamma_{b a} & -\frac{i}{2} u & \frac{i}{2} u \\
\frac{i}{2} u & -\frac{i}{2} u & s+\Gamma_{b a}-i(\Delta+\delta) & 0 \\
-\frac{i}{2} u & \frac{i}{2} u & 0 & s+\Gamma_{b a}+i(\Delta+\delta)
\end{array}\right]\left[\begin{array}{c}
P_{a a}(s) \\
P_{b b}(s) \\
P_{a b}(s) \\
P_{b a}(s)
\end{array}\right]=\left[\begin{array}{c}
\rho_{a a}(0)+\left(\gamma_{b a} / s\right) \\
\rho_{b b}(0) \\
\rho_{a b}(0) \\
\rho_{b a}(0)
\end{array}\right]
$$

where the Laplace transform $\mathbf{P}(s)$ of the density-matrix operator $\rho(t)$ is defined by

$$
\mathbf{P}(s)=\int_{0}^{\infty} \rho(t) e^{-s t} d t
$$

and $\rho(0)$ describes the state of the system at the initial time $t_{0}=0$. Equation (53) can be easily solved algebraically, and we present only the solution for the case where the system is initially in the ground level $|a\rangle$ :

$$
\begin{aligned}
& P_{b b}(s)=\frac{\gamma_{a b}\left[s+\Gamma_{b a}-i(\Delta+\delta)\right]\left[s+\Gamma_{b a}+i(\Delta+\delta)\right]+\frac{1}{2} u^{2}\left(s+\Gamma_{b a}\right)}{s \mathscr{D}(s)}, \\
& P_{a b}(s)=\frac{-\frac{i}{2} u\left(s-\gamma_{a b}+\gamma_{b a}\right)\left[s+\Gamma_{b a}+i(\Delta+\delta)\right]}{s \mathscr{D}(s)}, \\
& P_{b a}(s)=P_{a b}^{*}(s),
\end{aligned}
$$




$$
P_{a a}(s)=\frac{1}{s}-P_{b b}(s),
$$

where

$$
\mathscr{D}(s) \equiv\left(s+\gamma_{a b}+\gamma_{b a}\right)\left[s+\Gamma_{b a}-i(\Delta+\delta)\right]\left[s+\Gamma_{b a}+i(\Delta+\delta)\right]+u^{2}\left(s+\Gamma_{b a}\right) .
$$

In general, the inverse Laplace transform on $\mathbf{P}(s)$ is not easy to evaluate, but at the exact "shifted" resonance condition $\Delta+\delta=0$ one can readily carry it out and arrive at the following analytical expressions for the density-matrix elements:

$$
\begin{aligned}
\rho_{b b}(t)= & \frac{\gamma_{a b} \Gamma_{b a}+\frac{1}{2} u^{2}}{\left(\gamma_{a b}+\gamma_{b a}\right) \Gamma_{b a}+u^{2}}+\frac{\gamma_{a b}\left(s_{+}+\Gamma_{b a}\right)+\frac{1}{2} u^{2}}{2 s_{+}\left[\left(\gamma_{a b}+\gamma_{b a}-\Gamma_{b a}\right)^{2}-4 u^{2}\right]^{1 / 2}} \exp \left(s_{+} t\right) \\
& -\frac{\gamma_{a b}\left(s_{-}+\Gamma_{b a}\right)+\frac{1}{2} u^{2}}{2 s_{-}\left[\left(\gamma_{a b}+\gamma_{b a}-\Gamma_{b a}\right)^{2}-4 u^{2}\right]^{1 / 2}} \exp \left(s_{-} t\right), \\
\rho_{a b}(t)= & \frac{\frac{i}{2} u\left(\gamma_{a b}-\gamma_{b a}\right)}{\left(\gamma_{a b}+\gamma_{b a}\right) \Gamma_{b a}+u^{2}}+\frac{i}{2 s_{+}\left[\left(\gamma_{a b}+\gamma_{b a}-\Gamma_{b a}\right)^{2}-4 u^{2}\right]^{1 / 2}} \exp \left(s_{+} t\right) \\
& +\frac{\frac{i}{2} u\left[s_{-}+\left(\gamma_{b a}-\gamma_{a b}\right)\right]}{2 s_{-}\left[\left(\gamma_{a b}+\gamma_{b a}-\Gamma_{b a}\right)^{2}-4 u^{2}\right]^{1 / 2}} \exp \left(s_{-} t\right), \\
\rho_{b a}(t)= & \rho_{a b}^{*}(t),
\end{aligned}
$$

and

$$
\rho_{a a}(t)=1-\rho_{b b}(t)
$$

where

$$
s_{ \pm} \equiv \frac{1}{2}\left\{-\left(\gamma_{a b}+\gamma_{b a}+\Gamma_{b a}\right) \pm\left[\left(\gamma_{a b}+\gamma_{b a}-\Gamma_{b a}\right)^{2}-4 u^{2}\right]^{1 / 2}\right\}
$$

We note that both $s_{+}$and $s_{-}$possess negative real parts which are responsible for the damping of the oscillation of the density-matrix operator $\rho(t)$ when evolving in time and driven by the laser field. The steady-state solution of Eq. (44), not restricted to the resonance condition $\Delta+\delta=0$, also can be easily obtained,

$$
\begin{aligned}
& \bar{\rho}_{b b}=\frac{\gamma_{a b}\left[(\Delta+\delta)^{2}+\Gamma_{b a}^{2}\right]+\frac{1}{2} u^{2} \Gamma_{b a}}{\left(\gamma_{b a}+\gamma_{a b}\right)\left[(\Delta+\delta)^{2}+\Gamma_{b a}^{2}\right]+u^{2} \Gamma_{b a}}, \\
& \bar{\rho}_{a b}=\frac{\frac{1}{2} u\left(\gamma_{b a}-\gamma_{a b}\right)\left[(\Delta+\delta)-i \Gamma_{b a}\right]}{\left(\gamma_{b a}+\gamma_{a b}\right)\left[(\Delta+\delta)^{2}+\Gamma_{b a}^{2}\right]+u^{2} \Gamma_{b a}}, \\
& \bar{\rho}_{b a}=\bar{\rho}_{a b}^{*},
\end{aligned}
$$

and

$$
\bar{\rho}_{a a}=1-\bar{\rho}_{b b},
$$

which are independent of initial conditions and become time independent in the appropriate rotating frame defined in Eq. (51).

\section{MULTIPHOTON RESONANCE FLUORESCENCE SPECTRUM OF TWO-LEVEL SYSTEMS}

To elucidate the usefulness of the current approach, we present the first study of the multiphoton resonance fluorescence spectrum of an ensemble of two-level systems driven by polychromatic fields in a thermal bath undergoing purely radiative relaxation. The relevant quantity is the autocorrelation function ${ }^{31}$ of the transition dipole operators $\hat{d}^{\dagger} \equiv|b\rangle\langle a|$ and $\hat{d} \equiv|a\rangle\langle b|$, namely, $g\left(t ; t^{\prime}\right)=\left\langle\hat{d}^{\dagger}\left(t^{\prime}\right) \hat{d}(t)\right\rangle, t>t^{\prime}$, in normal order. The two-time average $g\left(t ; t^{\prime}\right)$ can be evaluated by the use of the quantum regression theorem ${ }^{10(d), 32}$ and expressed as

$$
g\left(t ; t^{\prime}\right)=\hat{U}_{b a ; a a}\left(t ; t^{\prime}\right) \hat{\rho}_{a b}\left(t^{\prime}\right)+\hat{U}_{b a ; b a}\left(t ; t^{\prime}\right) \hat{\rho}_{b b}\left(t^{\prime}\right),
$$

where $\hat{U}\left(t ; t^{\prime}\right)$ has been given in Eq. (30) and $\hat{\rho}(t)$ in Eq. (29). Explicitly, Eq. (68) can be written as

$$
g\left(t ; t^{\prime}\right)=\sum_{\{m\}} \sum_{\{n\}}\left(G_{1} G_{2}+G_{3} G_{4}\right),
$$


where

$$
\begin{aligned}
& G_{1}=\sum_{\mu, \nu} \sum_{\{k\}}\left\langle b a ;\{0\} \mid \Omega_{\mu v,\{k\}}\right\rangle\left\langle\Omega_{\mu v,\{k\}}^{*} \mid a a ;\{m\}\right\rangle \\
& \times\left\{\exp \left[-i \Omega_{\mu v,\{k\}}\left(t-t^{\prime}\right)\right]\right. \\
& \left.+\frac{\gamma_{b a}}{i \Omega_{\mu v,\{k-m\}}}\left[\exp \left(-i \sum_{j=1}^{M} m_{j} \omega_{j}\left(t-t^{\prime}\right)\right]-\exp \left[-i \Omega_{\mu v,\{k\}}\left(t-t^{\prime}\right)\right]\right]\right\} \exp \left[-i \sum_{j=1}^{M} m_{j} \omega_{j} t^{\prime}\right), \\
& G_{2}=\sum_{\mu, v\{k\}}\left\langle a b ;\{n\} \mid \Omega_{\mu v ;\{k\}}\right\rangle\left\langle\Omega_{\mu v,\{k\}}^{*} \mid a a ;\{0\}\right\rangle \\
& \times\left(\exp \left(-i \Omega_{\mu v,\{k\}} t^{\prime}\right)+\frac{\gamma_{b a}}{i \Omega_{\mu v,\{k\}}}\left[1-\exp \left(-i \Omega_{\mu v,\{k\}} t^{\prime}\right)\right]\right) \exp \left(i \sum_{j=1}^{M} n_{j} \omega_{j} t^{\prime}\right), \\
& G_{3}=\sum_{\mu, \nu} \sum_{\{k\}}\left\langle b a ;\{0\} \mid \Omega_{\mu v,\{k\}}\right\rangle\left\langle\Omega_{\mu v,\{k\}}^{*} \mid b a ;\{m\}\right\rangle \exp \left[-i \Omega_{\mu v,\{k\}}\left(t-t^{\prime}\right)\right] \exp \left[-i \sum_{j=1}^{M} m_{j} \omega_{j} t^{\prime}\right),
\end{aligned}
$$

and

$$
\begin{aligned}
G_{4}=\sum_{\mu, v\{k\}} \sum_{\{k} & \left\langle b b ;\{n\} \mid \Omega_{\mu v,\{k\}}\right\rangle\left\langle\Omega_{\mu v,\{k\}}^{*} \mid a a ;\{0\}\right\rangle \\
& \times\left[\exp \left(-i \Omega_{\mu v,\{k\}} t^{\prime}\right)+\frac{\gamma_{b a}}{i \Omega_{\mu v,\{k\}}}\left[1-\exp \left(-i \Omega_{\mu v,\{k\}} t^{\prime}\right)\right]\right) \exp \left(i \sum_{j=1}^{M} n_{j} \omega_{j} t^{\prime}\right),
\end{aligned}
$$

assuming the two-level system is initially in its ground level $|a\rangle$, i.e., $\hat{\rho}_{\alpha \beta}(0)=\delta_{\alpha a} \delta_{\beta a}$. The important feature here is the nonstationary nature of the correlation function $g\left(t ; t^{\prime}\right)$ even at some large time $t^{\prime}$, i.e., $g\left(t ; t^{\prime}\right)$ depends upon both the correlation time $\tau \equiv t-t^{\prime}$ and the starting time $t^{\prime}$. [Note that in the GVV, or RWA, limit, $g\left(t ; t^{\prime}\right) \rightarrow g(\tau)$, to be shown later, independent of $t^{\prime}$.] Thus it is a natural step here to bring up the notion of the time-dependent physical spectrum $^{22-26}$ which takes into account the true environment in which the detection of the scattering lights is performed. However, it is also possible to introduce a meaningful time-averaged scheme on the correlation function $g\left(t ; t^{\prime}\right)$ based on the fact that different two-level systems may have entered the laser fields at different times, e.g., in the atomic beam experiment, therefore rendering a meaningful power spectrum as in the exactly stationary case.

\section{A. Power spectrum-exact nonperturbative treatment}

We define the time-averaged correlation function as

$$
\bar{g}(\tau)=\lim _{T_{s} \rightarrow \infty}\left(\frac{1}{T_{s}} \int_{0}^{T_{s}} d t^{\prime} g\left(t ; t^{\prime}\right)\right)\left(t^{\prime} \rightarrow \infty\right),
$$

involving integrating $g\left(t ; t^{\prime}\right)$ over all possible times $t^{\prime}$, while keeping the correlation time $\tau \equiv t-t^{\prime}$ constant. The power spectrum $\bar{I}(\omega)$ corresponding to the time-averaged correlation function $\bar{g}(\tau)$ can then be evaluated via the Fourier transform:

$$
\bar{I}(\omega) \equiv \operatorname{Re}\left[\int_{0}^{\infty} d \tau e^{+i \omega \tau} \bar{g}(\tau)\right] .
$$

$\bar{I}(\omega)$ can be decomposed into a coherent part $\bar{I}_{\text {coh }}(\omega)$ and an incoherent part $\bar{I}_{\text {inc }}(\omega)$, namely,

$$
\begin{aligned}
\bar{I}_{\mathrm{coh}}(\omega)=-\pi \gamma_{b a}^{2} \operatorname{Re}\left\{\sum_{\{m\}}\right. & {\left.\left[\sum_{\mu, v} \sum_{\{k\}}\left\langle b a ;\{0\} \mid \Omega_{\mu v,\{k\}}\right\rangle\left\langle\Omega_{\mu v,\{k\}}^{*} \mid a a ;\{m\}\right\rangle \frac{1}{\Omega_{\mu v,\{k-m\}}} \delta \mid \omega-\sum_{i=1}^{M} m_{i} \omega_{i}\right]\right) } \\
\times & \left.\left.\times \sum_{\mu, \nu} \sum_{\{k\}}\left\langle a b ;\{m\} \mid \Omega_{\mu v,\{k\}}\right\rangle\left\langle\Omega_{\mu v,\{k\}}^{*} \mid a a ;\{0\}\right\rangle \frac{1}{\Omega_{\mu v,\{k\}}}\right]\right\}
\end{aligned}
$$

and

$$
\bar{I}_{\text {inc }}(\omega)=-\gamma_{b a} \operatorname{Re}\left[\sum_{\{m\}}(A B+C D)\right),
$$

where

$$
\begin{aligned}
A=\sum_{\mu, \nu} \sum_{\{k\}}\left\langle b a ;\{0\} \mid \Omega_{\mu v,\{k\}}\right\rangle\left\langle\Omega_{\mu v,\{k\}}^{*} \mid b a ;\{m\}\right\rangle \\
\times\left(\Omega_{\mu v,\{k\}}-\omega\right)^{-1},
\end{aligned}
$$




$$
\begin{aligned}
& B=\sum_{\mu, v} \sum_{\{k\}}\left\langle b b ;\{m\} \mid \Omega_{\mu v,\{k\}}\right\rangle \\
& \times\left\langle\Omega_{\mu v ;\{k\}}^{*} \mid a a ;\{0\}\right\rangle / \Omega_{\mu v ;\{k\}}, \\
& C=\sum_{\mu, v\{k\}} \sum_{i k}\left\langle b a ;\{0\} \mid \Omega_{\mu v,\{k\}}\right\rangle\left\langle\Omega_{\mu v,\{k\}}^{*} \mid a a ;\{m\}\right\rangle \\
& \times\left[1+i \gamma_{b a}\left(\Omega_{\mu v ;\{k-m\}}\right)^{-1}\right]\left(\Omega_{\mu v ;\{k\}}-\omega\right)^{-1},
\end{aligned}
$$

and

$$
\begin{aligned}
D=\sum_{\mu, v} \sum_{\{k\}}\left\langle a b ;\{m\} \mid \Omega_{\mu v,\{k\}}\right\rangle \\
\quad \times\left\langle\Omega_{\mu v,\{k\}}^{*} \mid a a ;\{0\}\right\rangle / \Omega_{\mu v,\{k\}} .
\end{aligned}
$$

Equation (72) shows that the coherently scattered light comprises not only the elastic components (Rayleigh scattering) at $\omega=\omega_{1}, \omega_{2}, \ldots, \omega_{M}$, but also various harmonic components at $\omega=\sum_{i=1}^{M} m_{i} \omega_{i}$, where $\sum_{i} m_{i}$ must be odd integers. Equation (73) shows that the incoherently scattered light contains frequency components at the positions defined by the denominator $\left(\Omega_{\mu v,\{k\}}-\omega\right)^{-1}$, where $\Sigma_{\{k\}} \equiv \Sigma_{i} k_{i}$ is an odd integer if $\mu=\nu$ and an even integer if $\mu \neq \nu$. Thus there exists in principle an infinite number of components, with the intensity of each component depending upon the degree of mixing of the two levels by the fields. In particular, for a one-mode laser field (with the frequency $\omega_{L}$ ), the coherently scattered light (of infinitesimal narrow width) can be detected at frequencies $\omega=\omega_{L}, 3 \omega_{L}, 5 \omega_{L}, \ldots$, and the incoherently scattered light (appearing generally in triplet) will appear also at $\omega \cong \omega_{L}, 3 \omega_{L}, 5 \omega_{L}, \ldots$. We emphasize that the expressions Eqs. (72) and (73) are general results applicable to arbitrary numbers and strengths of the fields.

\section{B. Power spectrum-GVV perturbative treatment}

In the one-mode case, when the resonance condition $\omega_{b a} \cong(2 n+1) \omega_{L}$, with $n=0,1,2, \ldots$, and the laser field is not very intense, we can make use of the GVVLiouvillian presented in Sec. IV to derive a completely stationary correlation function $g\left(t ; t^{\prime}\right)$ at some large $t^{\prime}$ and thus the corresponding power spectrum. We again employ the expression Eq. (68) to evaluate the required correlation function, but at the moment we assume the atom is in equilibrium with the laser field, i.e., where $\hat{U}\left(t-t^{\prime}\right)$, the GVV-superevolution operator, is to be derived from Eq. (44), and the steady-state quantities $\bar{\rho}_{a b}$ and $\bar{\rho}_{b b}$ have been given in Eqs. (64) and (65). Instead of obtaining an expression directly for the correlation function $g\left(t-t^{\prime}\right)$ in Eq. (74), we can first carry out the Laplace transform of $g\left(t-t^{\prime}\right)$, namely,

$$
G(s)=\int_{0}^{\infty} g(\tau) e^{-s \tau} d \tau,
$$

which gives the relation

$$
G(s)=\mathscr{U}_{b a ; a a}(s) \bar{\rho}_{a b}+\mathscr{U}_{b a ; b a}(s) \bar{\rho}_{b b},
$$

where

$$
\mathscr{U}_{b a ; a a}(s) \equiv \int_{0}^{\infty} \hat{U}_{b a ; a a}(\tau) e^{-\lrcorner \tau} d \tau
$$

and

$$
\mathscr{U}_{b a ; b a}(s) \equiv \int_{0}^{\infty} \hat{U}_{b a ; b a}(\tau) e^{-s \tau} d \tau
$$

Using Eq. (53), Eqs. (77) and (78) can be recast into analytical forms,

$$
\mathscr{U}_{b a ; a a}(s)=\frac{i \frac{u}{2}\left(s+\gamma_{b a}-\gamma_{a b}\right)\left[s+\Gamma_{b a}-i(\Delta+\delta)\right]}{s \mathscr{D}(s)}
$$

and

$$
\mathscr{U}_{b a ; b a}(s)=\frac{\left(s+\gamma_{a b}+\gamma_{b a}\right)\left[s+\Gamma_{b a}-i(\Delta+\delta)\right]+\frac{u^{2}}{2}}{\mathscr{D}(s)},
$$

where $\mathscr{D}(s)$ has been given in Eq. (59). The power spectrum corresponding to the correlation function given in Eq. (74) can thus be written as

$$
\bar{I}(\omega) \equiv \operatorname{Re}\left[\int_{0}^{\infty} g(\tau) e^{i\left[\omega-(2 n+1) \omega_{L}\right] \tau} d \tau\right],
$$

which contains a coherent part

$$
\bar{I}_{\text {coh }}(\omega)=\pi\left|\bar{\rho}_{a b}\right|^{2} \delta(v(\omega))
$$

and an incoherent part

$$
\bar{I}_{\text {inc }}(\omega)=\operatorname{Re}\left[\mathscr{U}_{b a ; b a}(-i v(\omega)) \bar{\rho}_{b b}+\mathscr{V}(-i v(\omega))\right] \text {, }
$$

where with

$$
\begin{aligned}
& v(\omega)=\omega-(2 n+1) \omega_{L}, \\
& k=\gamma_{a b}+\gamma_{b a},
\end{aligned}
$$

and

$$
z=\Gamma_{b a}-i(\Delta+\delta) .
$$

In the one-photon, i.e., $n=0, \mathrm{RWA}$ limit, the power spec- 
trum given in Eqs. (82) and (83) has been analyzed in great detail by Mollow. In the multiphoton, i.e., $n \neq 0$, resonance case, because of the close resemblance of the multiphoton GVV-Liouvillian $\hat{L}_{\mathrm{GVV}}$ in Eq. (45) to the RWA-Liouvillian, similar conclusions to those in the RWA limit ${ }^{9,10}$ hold, namely, (i) the coherent scattered light occurs at $\omega=(2 n+1) \omega_{L}$ and (ii) the incoherently scattered light spectrum consists of either one peak centered at $\omega=(2 n+1) \omega_{L}$ when the effective Rabi frequency $|u| \ll \gamma_{b a}$ or three peaks centered at $\omega=(2 n+1) \omega_{L}$ and separated by distance $|u|$ when $|u| \gg \gamma_{b a}$. In the pure radiative case, i.e., $\gamma_{a b}=0$ and $\Gamma_{b a}=\frac{1}{2} \gamma_{b a}$, the three peaks are symmetrical and approach integrated strengths of $\frac{1}{4}, \frac{1}{2}$, and $\frac{1}{4}$ and radiative linewidths of $\frac{3}{2} \gamma_{b a}, \gamma_{b a}$, and $\frac{3}{2} \gamma_{b a}$, respectively, when $|u| \gg \gamma_{b a}$.

\section{Time-dependent physical spectrum}

It has been shown ${ }^{25}$ that the time-dependent physical spectrum of any signal can be expressed as a twofold convolution integral over the time and frequency of a filter function and a time-dependent quasispectrum that depends on the signal only. We follow closely, apart from an overall constant, the procedure introduced by Nienhuis $^{25}$ of defining the time-dependent physical spectrum, namely,

$\bar{I}(\omega, t ; \Gamma) \equiv \int d \omega_{0} \int_{0}^{\infty} d \tau S\left(\omega_{0}, \tau ; \Gamma\right) I\left(\omega-\omega_{0}, t-\tau\right)$,

where the filter smoothing function $S\left(\omega_{0}, \tau ; \Gamma\right)$ takes the form

$$
S\left(\omega_{0}, \tau ; \Gamma\right)=\frac{1}{2 \pi} \Gamma^{2}\left[\left(\frac{1}{2} \Gamma\right)^{2}+\omega_{0}^{2}\right]^{-1} \exp (-\Gamma \tau),
$$

with $\Gamma$ denoting the filter width, and the quasispectrum (the Page-Lampard spectrum) $I(v, t)$ is defined as

$$
I(v, t)=\operatorname{Re}\left[\int_{-\infty}^{t} d t^{\prime} e^{\left.i v t-t^{\prime}\right)} g\left(t ; t^{\prime}\right)\right],
$$

with the correlation function given in Eq. (69). Assuming the laser fields are turned on abruptly at time $t=0$, combination of Eqs. (85) - (87) yields the integral

$$
\begin{gathered}
\bar{I}(\omega, t ; \Gamma)=\Gamma \operatorname{Re}\left[\int_{0}^{t} d t_{1} \exp \left[-\Gamma\left(t-t_{1}\right)\right]\right. \\
\quad \times \exp \left(i \omega t_{1}\right) \exp \left[-\frac{\Gamma}{2} t_{1}\right) \\
\times \int_{0}^{t_{1}} d t_{2} \exp \left(-i \omega t_{2}\right) \\
\left.\times \exp \left(\frac{\Gamma}{2} t_{2}\right) g\left(t_{1} ; t_{2}\right)\right],
\end{gathered}
$$

which can be carried out analytically. The result is
$\bar{I}(\omega, t ; \Gamma)$

$$
=\operatorname{Re}\left(\sum_{\{m\}} \sum_{\left\{m^{\prime}\right\}} \sum_{\mu, v} \sum_{\{k\}} \sum_{\mu^{\prime}, v^{\prime}} \sum_{\left\{k^{\prime}\right\}}\left(Q_{1}+Q_{2}+Q_{3}+Q_{4}\right)\right],
$$

where

$$
\begin{aligned}
& Q_{1}=\gamma_{b a}^{2} \Gamma C_{1} C_{2} B_{1}\left(A_{1}-A_{2}\right), \\
& Q_{2}=\gamma_{b a} \Gamma\left(C_{3} C_{2}+C_{4} C_{5}\right) B_{2}\left(A_{1}-A_{3}\right), \\
& Q_{3}=\gamma_{b a} \Gamma C_{1} C_{6} B_{3}\left(A_{4}-A_{2}\right),
\end{aligned}
$$

and

$$
Q_{4}=\Gamma\left(C_{3} C_{6}+C_{4} C_{7}\right) B_{4}\left(A_{4}-A_{3}\right),
$$

with

$$
\begin{aligned}
& A_{1}=\left\{\exp \left[-i\left(\sum_{j=1}^{M}\left(m_{j}-m_{j}^{\prime}\right) \omega_{j} t\right]\right]\right. \\
& -\exp (-\Gamma t)\} /\left[\Gamma-i \sum_{j=1}^{M}\left(m_{j}-m_{j}^{\prime}\right) \omega_{j}\right], \\
& A_{2}=\left\{\exp \left[-\frac{\Gamma}{2} t\right] \exp \left[i \mid \omega-\sum_{j=1}^{M} m_{j} \omega_{j}\right] t\right] \\
& -\exp (-\Gamma t)] /\left[\frac{\Gamma}{2}+i\left[\omega-\sum_{j=1}^{M} m_{j} \omega_{j}\right]\right], \\
& A_{3}=\left[\exp \left(-\frac{\Gamma}{2} t\right] \exp \left[i\left(\omega-\Omega_{\mu v,\{k\}}\right) t\right]\right. \\
& -\exp (-\Gamma t)] /\left[\frac{\Gamma}{2}+i\left(\omega-\Omega_{\mu v,\{k\}}\right)\right), \\
& A_{4}=\left\{\exp \left[-i\left(\Omega_{\mu^{\prime} v^{\prime} ;\left(k^{\prime}+m-m^{\prime}\right\}}^{\prime}\right) t\right]\right. \\
& -\exp (-\Gamma t)\} /\left[\Gamma-i\left(\Omega_{\mu^{\prime} v ;\left\{k^{\prime}+m-m^{\prime}\right\}}^{\prime}\right)\right], \\
& B_{1}=\left[\frac{\Gamma}{2}-i\left[\omega-\sum_{j=1}^{M} m_{j}^{\prime} \omega_{j}\right)\right]^{-1} \text {, } \\
& B_{2}=\left(\frac{\Gamma}{2}-i\left(\omega-\Omega_{\mu v,\left\{k-m+m^{\prime}\right\}}\right)\right]^{-1}, \\
& B_{3}=\left(\frac{\Gamma}{2}-i\left(\omega+\Omega_{\mu^{\prime} v^{\prime} ;\left\{k^{\prime}-m^{\prime}\right\}}^{\prime}\right)\right)^{-1} \text {, } \\
& B_{4}=\left(\frac{\Gamma}{2}-i\left(\omega-\Omega_{\mu v,\{k-m\}}+\Omega_{\mu^{\prime} v,\left\{k^{\prime}-m^{\prime}\right\}}^{\prime}\right)\right]^{-1}, \\
& C_{1}=\left\langle b a ;\{0\} \mid \Omega_{\mu v,\{k\}}\right\rangle \\
& \times\left\langle\Omega_{\mu v,\{k\}}^{*} \mid a a ;\{m\}\right\rangle /\left(i \Omega_{\mu v ;\{k-m\}}\right), \\
& C_{2}=\left\langle a b ;\left\{m^{\prime}\right\} \mid \Omega_{\mu^{\prime} v^{\prime} ;\left\{k^{\prime}\right\}}^{\prime}\right\rangle \\
& \times\left\langle\Omega_{\mu^{\prime} v^{\prime} ;\left\{k^{\prime}\right\}}^{*} \mid a a ;\{0\}\right\rangle /\left(i \Omega_{\left.\mu^{\prime} v^{\prime} ; k^{\prime}\right\}}^{\prime}\right),
\end{aligned}
$$




$$
\begin{aligned}
C_{3}= & \left\langle b a ;\{0\} \mid \Omega_{\mu v,\{k\}}\right\rangle \\
& \left.\times\left\langle\Omega_{\mu v,\{k\}}^{*} \mid a a ;\{m\}\right\rangle \mid 1-\frac{\gamma_{b a}}{i \Omega_{\mu v,\{k-m\}}}\right), \\
C_{4}= & \left\langle b a ;\{0\} \mid \Omega_{\mu v,\{k\}}\right\rangle\left\langle\Omega_{\mu \nu,\{k\}}^{*} \mid b a ;\{m\}\right\rangle, \\
C_{5}= & \left\langle b b ;\left\{m^{\prime}\right\} \mid \Omega_{\mu^{\prime} v^{\prime} ;\left\{k^{\prime}\right\}}^{\prime}\right\rangle \\
& \times\left\langle\Omega_{\mu^{\prime} v^{\prime} ;\left\{k^{\prime}\right\}}^{\prime} \mid a a ;\{0\}\right\rangle /\left(i \Omega_{\mu^{\prime} v^{\prime},\left\{k^{\prime}\right\}}^{\prime}\right), \\
C_{6}= & \left\langle a b ;\left\{m^{\prime}\right\} \mid \Omega_{\mu^{\prime} v^{\prime} ;\left\{k^{\prime}\right\}}^{\prime}\right\rangle \\
& \left.\times\left\langle\Omega_{\mu^{\prime} v^{\prime} ;\left\{k^{\prime}\right\}}^{*} \mid a a ;\{0\}\right\rangle \mid 1-\frac{\gamma_{b a}}{i \Omega_{\mu^{\prime} v^{\prime} ;\left\{k^{\prime}\right\}}^{\prime}}\right),
\end{aligned}
$$

and

$$
\begin{aligned}
C_{7}= & \left\langle b b ;\left\{m^{\prime}\right\} \mid \Omega_{\mu^{\prime} v^{\prime} ;\left\{k^{\prime}\right\}}^{\prime}\right\rangle \\
& \times\left\langle\Omega_{\left.\mu^{\prime} v^{\prime} ; k^{\prime}\right\}}^{\prime *} \mid a a ;\{0\}\right\rangle\left(1-\frac{\gamma_{b a}}{i \Omega_{\left.\mu^{\prime} v^{\prime} ; k^{\prime}\right\}}^{\prime}}\right) .
\end{aligned}
$$

We note the following. (i) For the single- mode case, when the laser frequency $\omega_{L}$ is close to $\omega_{b a}=E_{b}-E_{a}$, Eq. (89) reduces to that of Eberly et al. ${ }^{26(a)}$ in the RWA limit. This can be easily seen first using Eq. (44) via (48) by neglecting antirotating terms, i.e., $\delta=0$ and $\frac{1}{2} u=b$, and then invoking Eqs. (68) and (88). (ii) In the limit $t \rightarrow \infty$ and $\Gamma \rightarrow 0, \bar{I}(\omega, t ; \Gamma)$ reduces to the time-averaged power spectrum $\bar{I}(\omega)$ given by Eqs. (72) and (73).

\section{CASE STUDY OF MULTIPHOTON RESONANCE FLUORESCENCE-RESULTS AND DISCUSSION}

We consider a closed system of an ensemble of twolevel atoms driven by a strong linearly polarized monochromatic field of frequency $\omega_{L}$. The two levels are of opposite parity with separation $\omega_{b a}=E_{b}-E_{a} \simeq 100.0$ (arbitrary units). Only radiative damping is considered. The spontaneous decay rate from the upper level $|b\rangle$ to the ground level $|a\rangle$ is $\gamma_{b a}$ and the (half-)Rabi frequency characterizing the interaction strength of the atom with the field is $V_{a b}=\frac{1}{2}|\langle a|\mu \cdot \varepsilon| b\rangle|$. Both strong field and medium field results will be presented.

\section{A. Strong-field-case (exact) results: $\gamma_{a b}=1.0$ and $V_{a b}=25.0$ (arbitrary units)}

In order to demonstrate the strong field effects, here we have assumed a rather large spontaneous decay rate and a very strong strength when compared with the magnitude of the energy level difference of the two levels.

\section{Quasienergies, supereigenvalues,} and long time-averaged populations and coherences

There are intimate relationships between quasienergies $\lambda_{\alpha m}$, supereigenvalues $\Omega_{\alpha \beta ; m}$, and the long time-averaged density-matrix operator $\widehat{\rho}$. In Figs. 2(a) and 2(b), $\lambda_{\alpha m}$ and $\Omega_{\alpha \beta ; m}$ are shown as functions of the laser frequency $\omega_{L}$. These eigenvalues are obtained by numerically diagonalizing truncated, but converged, up to the five-photon matrices $\hat{L}_{F}$ in Eq. (17) and $\hat{H}_{F}$ in Eq. (36), respectively. Ex- plicitly speaking, the truncated matrix $\hat{L}_{F}$ is a $44 \times 44$ matrix [the Floquet-Liouvillian $\hat{L}_{F}$ diagonalized has been transformed to a one-photon rotating frame, cf. Eq. (51), and only the parts coupled together are retained; therefore, each photon block is a $4 \times 4$ submatrix], while the truncated $\hat{H}_{F}$ is a $22 \times 22$ matrix (a $2 \times 2$ submatrix for each photon block in ordinary Floquet basis [cf. Ref. 29(a)]). The relationships between $\lambda$ and $\Omega$ are given by Eq. (40): the pattern of avoided crossings, the periodic structures when varying from one photon block to another (cf. the indices on the right-hand sides of the figures), and distances between avoided crossing levels. Furthermore, the dashed squares shown in Fig. 2(b) show the strong mixed regions of the two levels caused by one-, three-, and five-photon resonance transitions, respectively. The splittings and stretches of the avoided crossing regions indicate the widths of the corresponding line shapes of the long time-averaged excitation spectrum $\bar{\rho}_{b b}$, as a function of $\omega_{L}$, depicted in Fig. 3(a). Another important feature of the $\lambda$ and $\Omega$ figures is that they display the shift of the
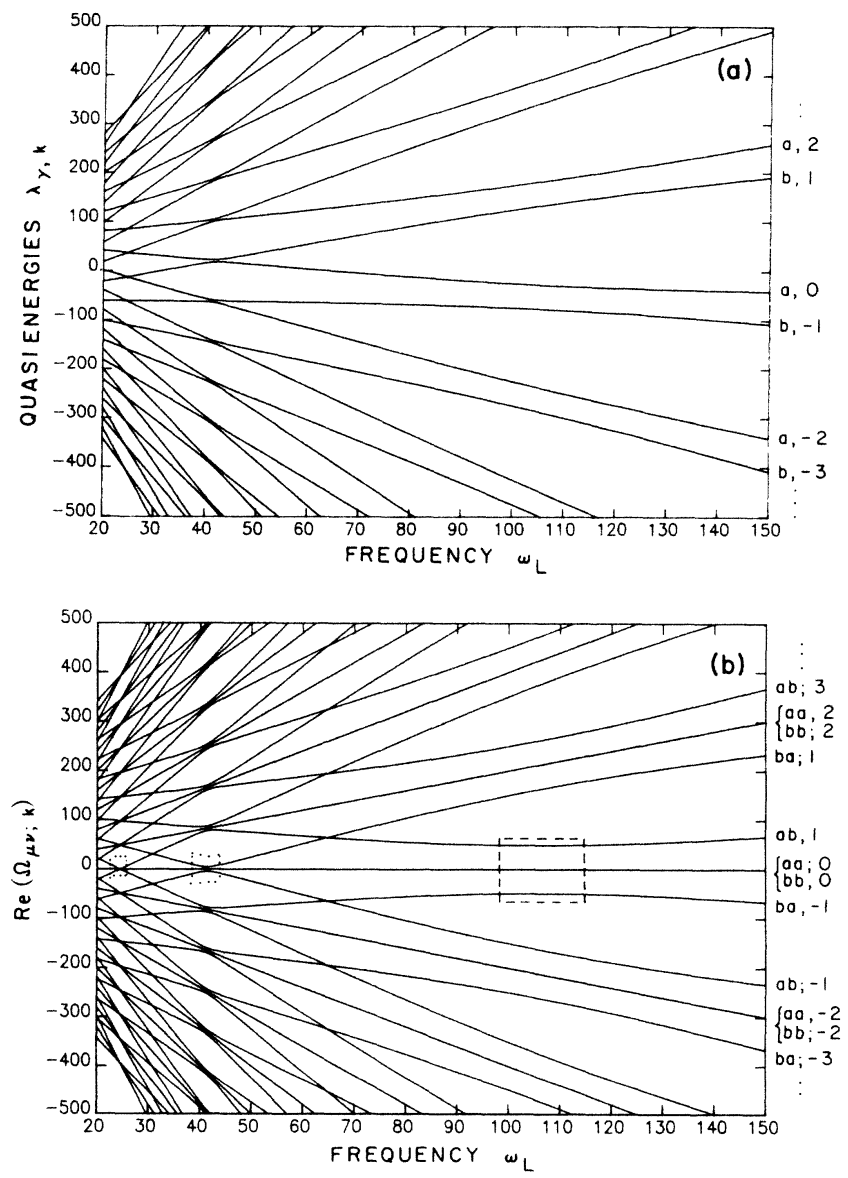

FIG. 2. (a) Quasienergies ( $\lambda$ 's) and (b) supereigenvalues $(\operatorname{Re} \Omega$ 's), for a closed system of two-level atoms driven by a monochromatic field of frequency $\omega_{L}$. Parameters used are $\omega_{b a}=100.0, \gamma_{b a}=1.0, \gamma_{a b}=0.0,\left|V_{a b}^{(1)}\right|=25.0$, and $\phi^{(1)}=0.0(\mathrm{ar}-$ bitrary units). Note that $\Omega_{a a ; k}$ and $\Omega_{b b ; k}$ are almost degenerate and cannot be distinguished in the graph. 
resonance transitions away from the natural frequency $\omega_{b a}$, i.e., strong resonances occur at frequencies other than those given by the equality $\omega_{b a}=(2 n+1) \omega_{L}$, with $n=0,1,2, \ldots$. This is most clearly seen in Figs. 3(a) and 3(b), the long time-averaged population $\bar{\rho}_{b b}$ and coherence $\bar{\rho}_{a b}$. In this case the one-, three-, and five-photon resonances have been shifted, respectively, from 100.0, 33.333, and 20.0 to $106.335,41.295$, and 24.525 [cf. Eq. (47)]. The width of the individual resonance line shape is approximately proportional to $\left\{V_{a b}^{2 n+1} /\left[\left(2 \omega_{L}\right)^{2 n}(n !)^{2}\right]\right\}$ given by Eq. (48). Also shown in Figs. 3(a) and 3(b) are the counterparts of $\bar{\rho}_{b b}$ and $\bar{\rho}_{a b}$ in the limit of $\gamma_{b a}=0$, indicating that the degree of the excitation caused by the external field may be substantially suppressed by the existence of the spontaneous decay from the upper level. This suppression is more pronounced for the higher-order photon resonance due to the decreasing effective coupling [cf. Eq. (48)]. We note that in this very strong field case, the GVV perturbative results (not shown) do not give good agreement when compared with the numerically exact results reported above.
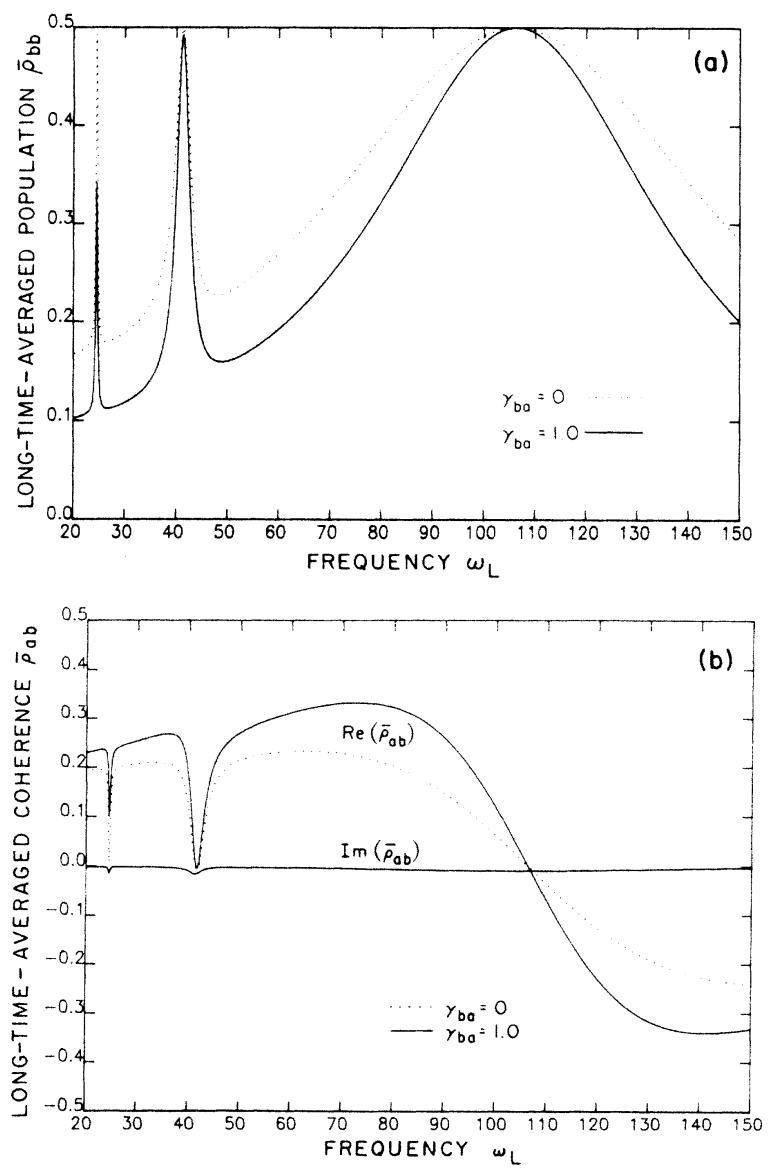

FIG. 3. (a) Long time-averaged population $\bar{\rho}_{b b}$ of the level $|b\rangle$ and (b) coherence $\bar{\rho}_{a b}$ between the two levels for a closed system of two-level atoms driven by a monochromatic field of frequency $\omega_{L}$. Parameters used are the same as those in Fig. 2. The one-, three-, and five-photon resonances (solid curves) occur at $\omega_{L}=106.335,41.295$, and 24.525 , respectively. Also shown are results for the nondamping $\left(\gamma_{b a}=0.0\right)$ case (dotted curves).

\section{Time evolution of the density-matrix operator}

Temporal distribution of the populations and coherences of an ensemble of systems reveals details of dressed atomic structure in the presence of strong fields, e.g., via the study of free-induction decay, and also dictates the character of the light waves generated during the scattering by atoms. In Figs. 4-6 we report the time-dependent behavior of both the population $\hat{\rho}_{b b}(t)$ and the coherence $\hat{\rho}_{a b}(t)$ at the shifted one-, three-, and five-photon resonances, i.e., at $\omega_{L}=106.335,41.295$, and 24.525 , respectively. There is a 1:1 correspondence between the characteristic periods of the oscillation of $\hat{\rho}_{b b}(t)$ and $\hat{\rho}_{a b}(t)$ and the quasienergy structure $\lambda\left(\omega_{L}\right)$ in Fig. 2(a) or the supereigenvalues $\Omega\left(\omega_{L}\right)$ in Fig. 2(b). The dominant characteristic period in the one-photon resonance case is caused by the oscillation between the two adjacent quasienergy levels indicated by the Floquet indices $(a, m)$ and $(b, m-1)$, appearing on the right-hand side of Fig. 2(a). The separation between these two levels approaches the Rabi frequency $2 V_{a b}$ at the weak field limit. The secondary characteristic period is dictated by the quasienergy differences $\left(\lambda_{b 1}-\lambda_{a 0}\right),\left(\lambda_{b,-1}-\lambda_{a,-2}\right), \ldots$ which introduce small wiggles on the predominant structure. The large spontaneous decay rate from level $|b\rangle$ to level $|a\rangle$ quickly suppresses the oscillation of $\hat{\rho}(t)$, centered at its time-averaged values, cf. Fig. 3(a), after the system flipflops between the two levels about 30 times. We note that the coherence $\hat{\rho}_{a b}(t)$ for each of the one-, three-, and fivephoton cases has been transformed into an individual appropriate rotating reference frame according to Eq. (51).

The three-photon resonances $\hat{\rho}_{b b}(t)$ and $\hat{\rho}_{a b}(t)$ are shown in Figs. 5(a)-5(c). The time dependence of these two quantities is now predominantly determined by the values $\left(\lambda_{a 0}-\lambda_{b,-3}\right),\left(\lambda_{a 2}-\lambda_{b,-1}\right), \ldots$ and modified by the differences $\left(\lambda_{b 1}-\lambda_{a 0}\right),\left(\lambda_{a 2}-\lambda_{b,-3}\right), \ldots$. Because in this case it takes longer, cf. the big hump in Fig. 5, to pump the system going up and down between levels $b$ and $a$ than in the one-photon case, the oscillations of $\hat{\rho}_{b b}(t)$ and $\hat{\rho}_{a b}(t)$ are not quite overpowered by the spontaneous decay damping, as in the one-photon case (cf. Fig. 4).

In Figs. 6(a)-6(c) we depict the time dependence of $\hat{\rho}_{b b}(t)$ and $\hat{\rho}_{a b}(t)$ at the shifted five-photon resonance. In this case the dominant oscillation is dictated by the quasienergy differences $\left(\lambda_{a 0}-\lambda_{b,-5}\right),\left(\lambda_{a 2}-\lambda_{b,-3}\right), \ldots$. Corresponding to a large flip-flopping period (beyond the figure) of the two levels, while less important oscillations due to differences $\left(\lambda_{a 0}-\lambda_{b,-3}\right),\left(\lambda_{a 2}-\lambda_{b,-5}\right), \ldots$ survive almost completely from the damping caused by the spontaneous decay. It is striking to note that even though the system has reached its steady state, it still oscillates vigorously with large amplitude.

\section{Time-averaged multiphoton resonance fluorescence: Power spectrum}

Resonance fluorescence scattering by atoms in the presence of strong laser fields is a delicate nonlinear process at least in two aspects: (i) It is a cascade process via an infinite number of dressed atomic states and (ii) it requires strong resonance mixings, either by one photon or by 

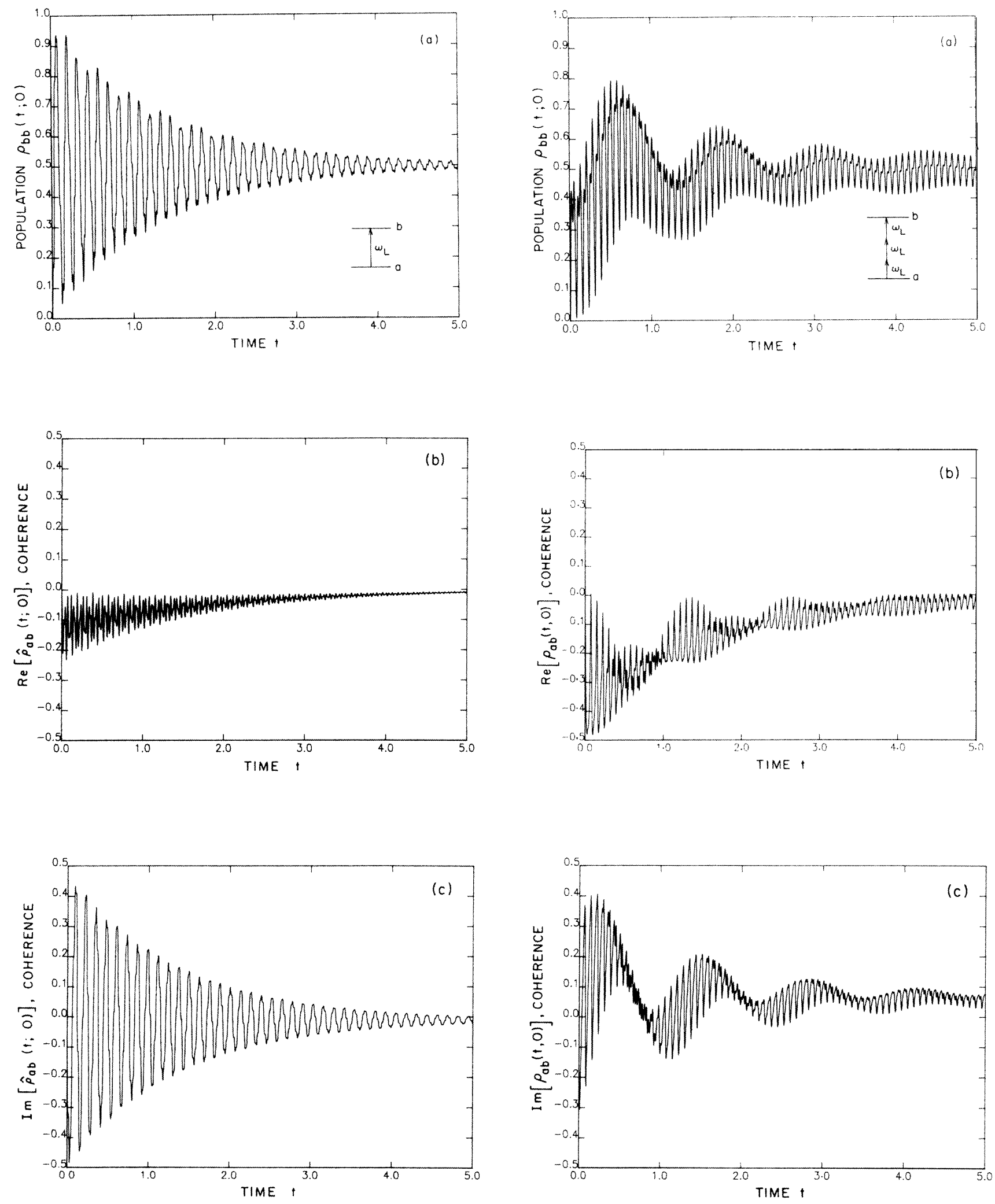

FIG. 4. (a) Time-dependent population $\hat{\rho}_{b b}(t)$ and (b) and (c) coherence $\hat{\rho}_{a b}(t)$ at the one-photon resonance frequency $\omega_{L}=106.335$ (cf. Fig. 3). Parameters used are the same as in Fig. 2. The results are calculated in the one-photon rotating frame, see Eq. (51), $n=0$, assuming each atom is initially in its ground level $|a\rangle$.

FIG. 5. (a) Time-dependent population $\hat{\rho}_{b b}(t)$ and (b) and (c) coherence $\hat{\rho}_{a b}(t)$ at the three-photon resonance frequency $\omega_{L}=41.295$ (cf. Fig. 3). Parameters used are the same as in Fig. 2. The results are calculated in the three-photon rotating frame, see Eq. (51), $n=1$, assuming each atom is initially in its ground level $|a\rangle$. 
several photons, between unperturbed atomic levels. The strong mixings of levels produce sidebands, due to the ac Stark effect, in addition to those corresponding to the natural transition frequencies. The resonance fluorescence processes are schematically depicted in Fig. 7, where each doublet is characterized by a splitting $u$ between a pair of nearly degenerate quasienergy levels, and arrows indicate fluorescence cascade patterns. At each resonance condi-
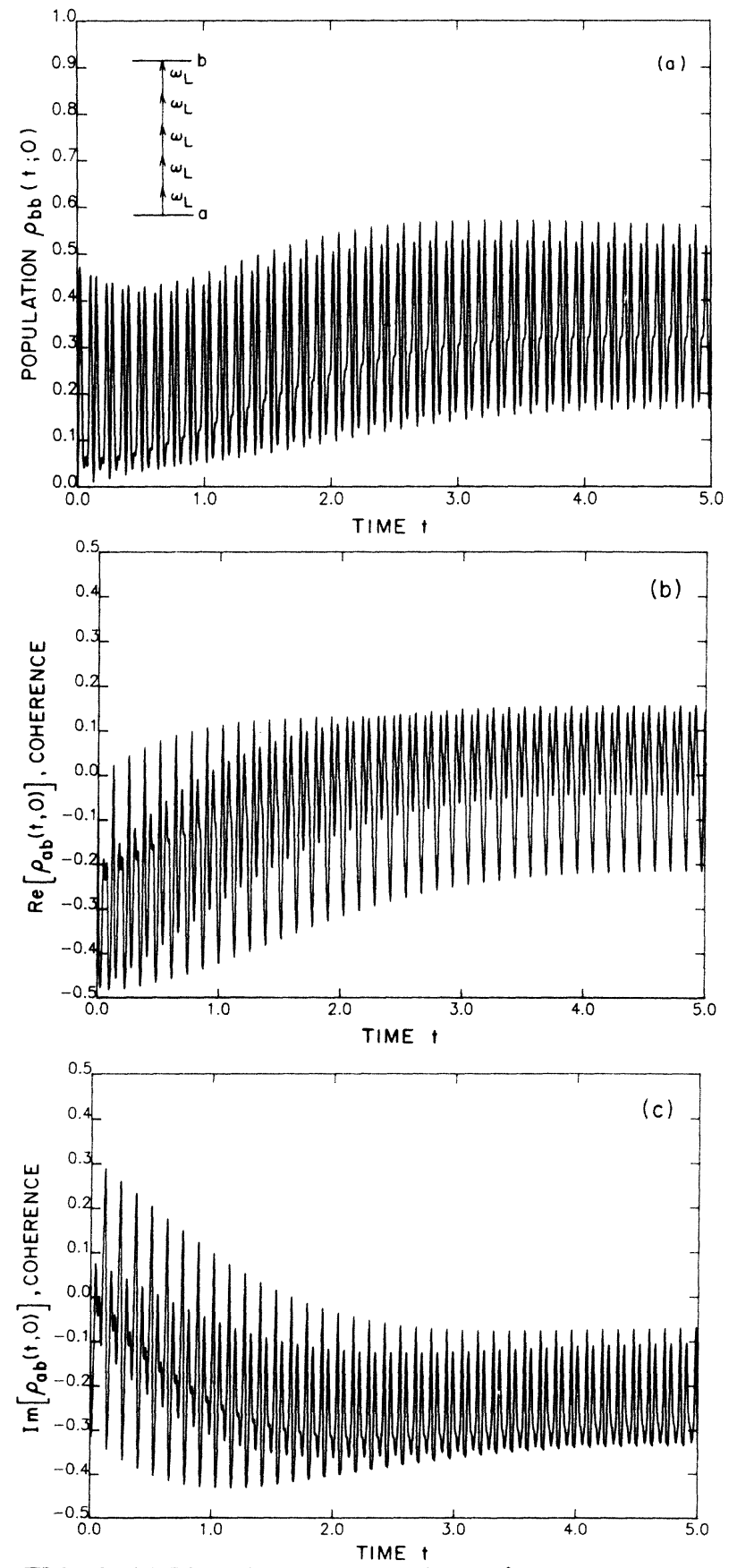

FIG. 6. (a) Time-dependent population $\hat{\rho}_{b b}(t)$ and (b) and (c) coherence $\hat{\rho}_{a b}(t)$ at the five-photon resonance frequency $\omega_{L}=24.525$ (cf. Fig. 3). Parameters used are the same as in Fig. 2. The results are calculated in the five-photon rotating frame, see Eq. (51), $n=2$, assuming each atom is initially in its ground level $|a\rangle$.

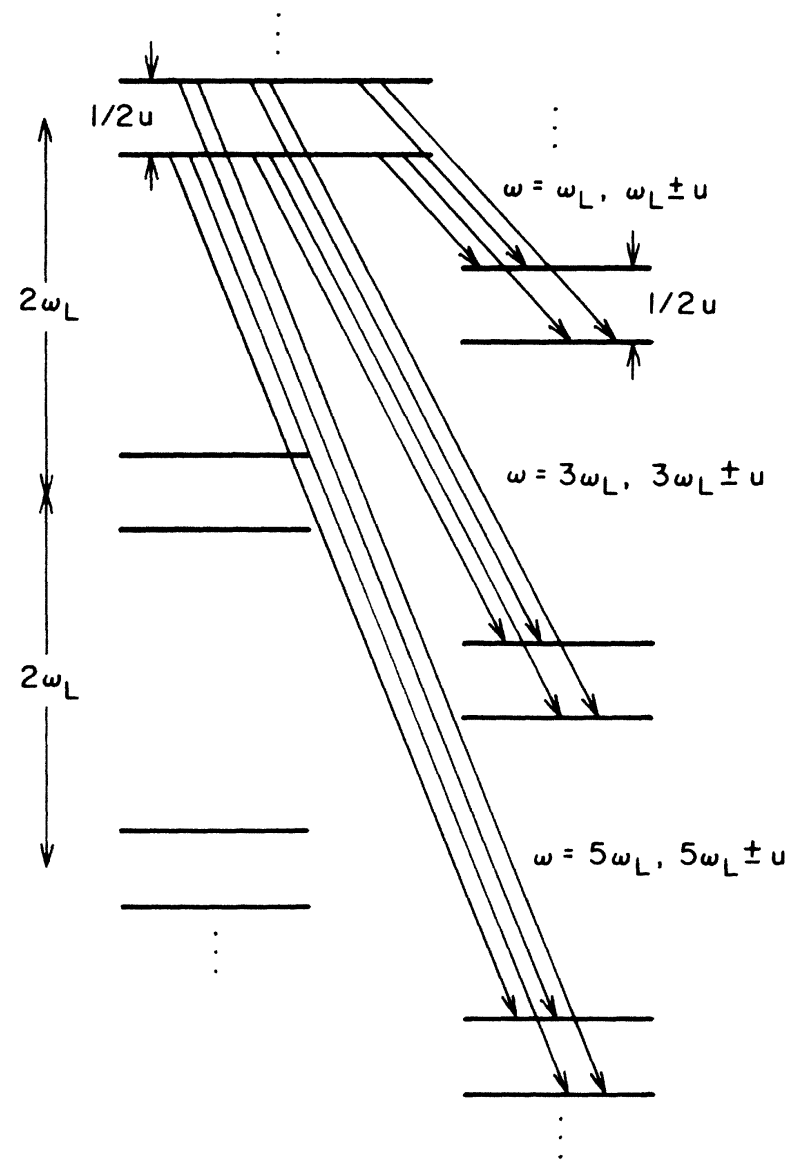

FIG. 7. Schematic cascade fluorescence processes of twolevel atoms driven by a monochromatic field of frequency $\omega_{L}$. The splitting $u$ of the doublets in each column is the splitting of the adjacent quasienergy levels in fig. 2(a) and is caused by the ac Stark effect and possible detuning $\Delta \equiv \omega_{b a}-(2 n+1) \omega_{L}$ at nearly resonant conditions. Each column is a collection of quasienergy levels of like parity; quasienergy levels belonging to different columns are of opposite parity. Arrows indicate parts of cascade fluorescence down the infinite number of quasienergy levels.

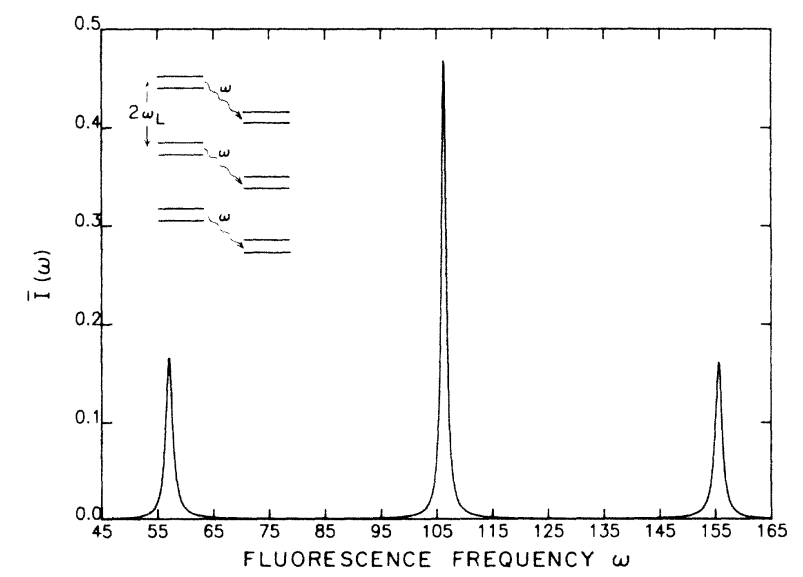

FIG. 8. Fluorescence spectra $\bar{I}(\omega)=\bar{I}(\omega, t ; \Gamma \rightarrow 0)$, Eq. (73), near $\omega \cong \omega_{L}$ for a system of two-level atoms driven by a monochromatic field, $\omega_{L}$ tuned at the shifted one-photon resonance $\omega_{L}=106.335$. Parameters given in Fig. 2. The inset shows the schematic cascade diagram (not to scale) (cf. Fig. 7). 
tion $\omega_{b a} \cong(2 n+1) \omega_{L}, \quad n=0,1,2, \ldots$, the most intense fluorescence light occurs around $\omega=(2 n+1) \omega_{L}$ and shows a triplet pattern. It is instructive to note that if we cut a cross section out of the supereigenvalue profile in Fig. 2(b) at a certain laser frequency $\omega_{L}$, we would see the intersection curves give the absolute positions of the spectral lines which are indeed infinite in number. However, only at the pseudocrossing regions can significant fluorescence lights be observed.

Figure 8 shows the fluorescence power spectrum, Eq.
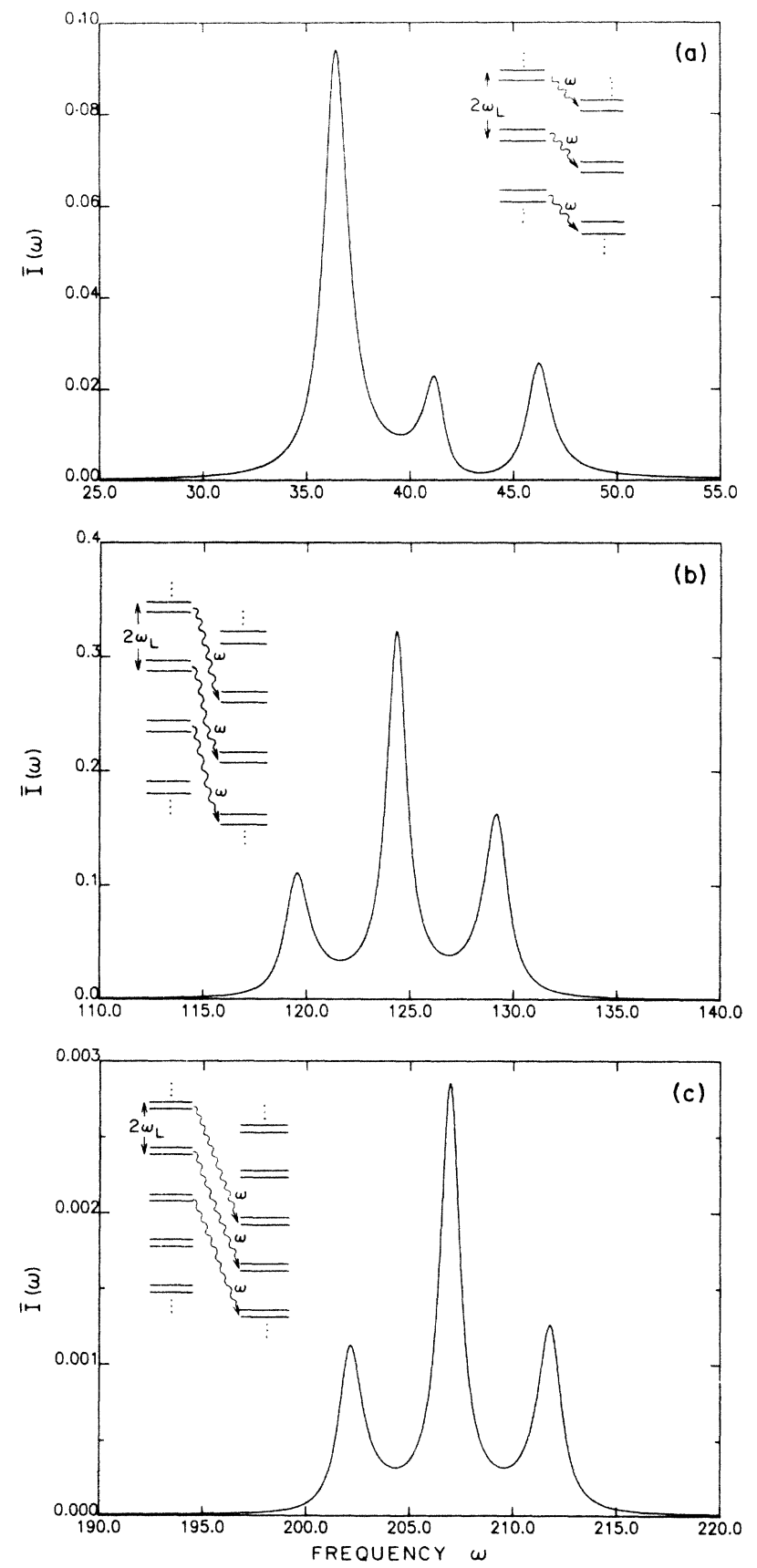

FIG. 9. Fluorescence spectra $\bar{I}(\omega)$, Eq. (73), near (a) $\omega \simeq \omega_{L}$, (b) $\omega \simeq 3 \omega_{L}$, and (c) $\omega \simeq 5 \omega_{L}$ for a system of two-level atoms driven by a monochromatic field, $\omega_{L}$ tuned at the shifted threephoton resonance $\omega_{L}=41.295$. Parameters given in Fig. 2. The inset in each figure shows the schematic cascade diagram (not to scale) (cf. Fig. 7).

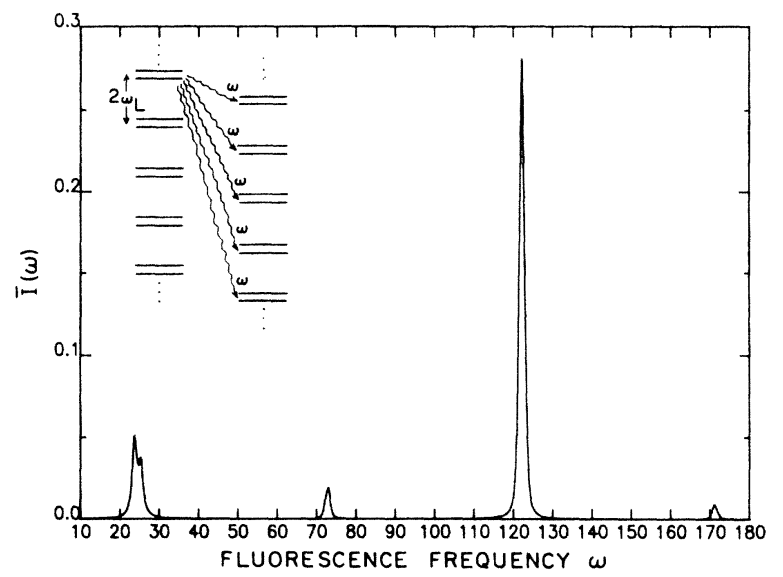

FIG. 10. Fluorescence spectra $\bar{I}(\omega)$, Eq. (73), near $\omega \cong \omega_{L}$, $3 \omega_{L}, 5 \omega_{L}$, and $7 \omega_{L}$ for a system of two-level atoms driven by a monochromatic field, $\omega_{L}$ tuned at the shifted five-photon resonance $\omega_{L}=24.525$. Parameters given in Fig. 2. The inset shows the schematic diagram (not to scale) (cf. Fig. 7).

(73), at the shifted one-photon resonance frequency, i.e., $\omega_{L}=106.335$, along with a schematic cascade diagram, similar to that of Fig. 7. The pattern of the three-peak spectrum, centered at $\omega_{L}=106.335$, is almost the same as that first obtained by Mollow ${ }^{9}$ in the RWA: The ratios of heights and widths are, respectively, about 1:3:1 and 3:2:3 as predicted by the RWA calculations. The sidebands are separated from the central peak by an amount approximately equal to $u=2 V_{a b}=50.0$. Other fluorescence triplets (not shown) at $3 \omega_{L}, 5 \omega_{L}, \ldots$ are too small to be detectable.

Figures 9 and 10 show the first results obtained for intense field multiphoton resonance-induced fluorescence spectra. At the three-photon shifted resonance frequency, i.e., $\omega_{L}=41.295$, we report strong triplet fluorescence spectra at two locations $\omega \approx \omega_{L}$ and $3 \omega_{L}$ [Figs. 9(a) and 9(b)] as well as a much weaker triplet around $\omega \approx 5 \omega_{L}$ [Fig. 9(c)]. Particularly interesting is the strongly asymmetric three-peak structure nearby $\omega \approx \omega_{L}$. This asymmetry can be largely attributed to the strong mixings not only among the resonant, or nearly resonant, unperturbed tetradic-Floquet states, e.g., $|a a ; 0\rangle,|b b ; 0\rangle,|a b ;+3\rangle$, and $|b a ;-3\rangle$, but also of the nonresonant states,e.g., $|a b ;+1\rangle,|b a ;-1\rangle, \ldots$. At a much weaker field (to be seen later), only those nearly resonant states are mixed, thus intense fluorescence light can only be observed at $3 \omega_{L}$ and possesses a symmetric triple-peak appearance. The intense fluorescence light and its asymmetric outlook at $\omega_{L}$ in this three-photon resonance case are really strong field effects.

In Fig. 10 we present the fluorescence power spectrum at the shifted five-photon resonance frequency $\omega_{L}=24.525$. A series of single peaks appearing at the frequencies $\omega_{L}, 3 \omega_{L}, 5 \omega_{L}, 7 \omega_{L}, \ldots$, is seen with the strongest one at $5 \omega_{L}$. In this case the splitting $u$ at the pseudocrossing region is very small compared with the spontaneous decay rate $\gamma_{b a}=1.0$ so that the would-be triplet pattern is obscured.

From Figs. 8-10, we can conclude a general rule concerning the intensity of the fluorescence lights: When the 
laser field is intense enough to induce multiphoton resonances [say $\omega_{b a} \cong(2 n+1) \omega_{L}$ ], the dominant fluorescence component always occurs at $\omega \cong(2 n+1) \omega_{L}$. Components in the lower-frequency side of the predominant one can have comparable intensities and often exhibit large asymmetry in the three-peak structure, while components in the higher-frequency side usually decrease rapidly in intensity as the harmonic order increases.

\section{Time-dependent physical spectrum}

Eberly et al. ${ }^{26(a), 26(b)}$ have presented careful examinations on time-dependent physical spectra at the onephoton resonance of two-level systems by assuming sudden switch-on and switch-off of the external field. Here we shall concentrate on the time-dependent physical spectra at the three-photon resonance of two-level systems by suddenly switching on the laser field. Making use of Eq. (89) and assuming the filter width $\Gamma=0.1$, we show the time-dependent physical spectrum $\bar{I}(\omega, t ; \Gamma=0.1)$ at $\omega_{L}=41.295$ and at $t=2.5,5.0,10.0$, and 30.0 in Figs. 11(a) and 11(b). By increasing the time $t$, we see that the
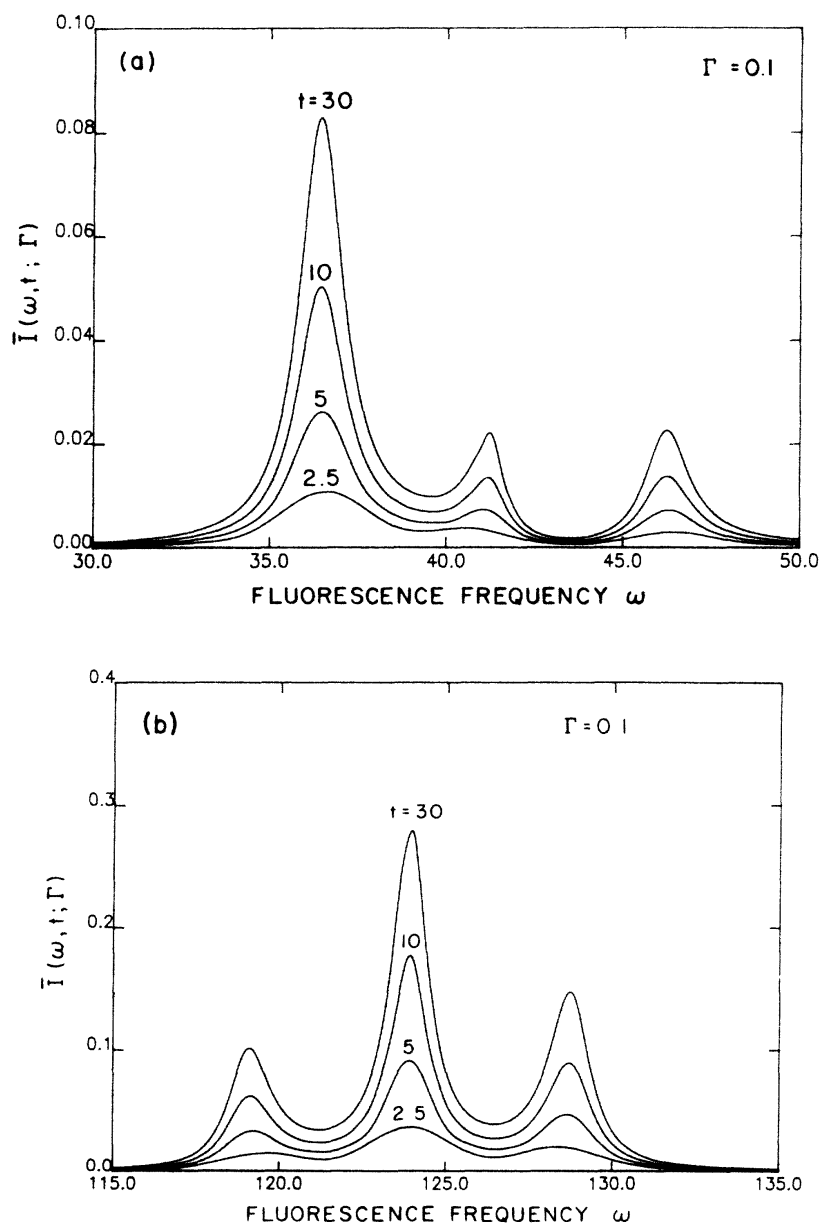

FIG. 11. Time-dependent physical spectra $\bar{I}(\omega, t ; \Gamma)$, Eq. (89), near (a) $\omega \cong \omega_{L}$ and (b) $\omega \cong 3 \omega_{L}$ for a system of two-level atoms driven by a monochromatic field, $\omega_{L}$ tuned at the shifted three-photon resonance $\omega_{L}=41.295$. The filter width is $\Gamma=0.1$, assuming the field is abruptly turned on at the time $t=0$. Parameters given in Fig. 2. three-peak structures, both at $\omega=\omega_{L}$ and $3 \omega_{L}$, gradually grow from zero and converge to the steady-state line shapes, very close to the ones shown in Figs. 9(a) and 9(b), except that each peak now is further broadened by an amount $\Gamma / 2$ [cf. constant $B$ 's in Eq. (89)] and is somewhat lowered down.

\section{B. Medium field cases}

In the first case (Sec. VI A), we have discussed results for a very strong field situation where nonperturbative calculations must be employed. In the following we shall present somewhat weaker field cases where the perturbative procedure, namely, GVV nearly degenerate perturbation theory, provides accurate results. The first medium field case will compare the GVV and RWA calculations at one-photon resonance, whereas the second medium field case is devoted to the GVV results at three-photon resonance. In both cases we are especially interested in the fluorescence power spectrum, i.e., Eq. (83).

$$
\begin{aligned}
& \text { 1. One-photon resonance case: } \\
& \gamma_{b a}=1.0 \text { and }\left|V_{a b}\right|=10.0 \text { (arbitrary units) }
\end{aligned}
$$

In Fig. 12(a) we present the GVV and RWA results for the long time-averaged population $\bar{\rho}_{b b}$ as a function of laser frequency $\omega_{L}$ : While the RWA results peak at the natural frequency $\omega_{b a}=100.0$, the GVV ones peak at a shifted resonance frequency $\omega_{L}^{(s)}=100.995$ which reveals the Bloch-Siegert shift due to the antirotating terms. We note that the RWA calculation is equivalent to a firstorder GVV perturbation calculation, i.e., dropping all correction terms of higher than the first order in Eq. (45). It is obvious that the width of the power-broadened onephoton resonance peak is one order of magnitude larger than the corresponding Bloch-Siegert shift, so that the more accurate GVV resonance profile is slightly displaced away from the RWA resonance profile. In Figs. 12(b), 12(c), and 12(d) we depict the fluorescence power spectra $\bar{I}(\omega)$, i.e., Eq. (83), at three different laser frequencies $\omega_{L}=100.0,90.0$, and 110.0. It is seen that nearby the resonance frequency, i.e., $\omega_{L} \approx 100.0$, the triplet spectra of the GVV and the RWA calculations agree with each other almost perfectly, cf. Fig. 12(b), while away from the resonance frequency, e.g., $\omega_{L}=100.0 \pm 10.0$, the two calculations show large differences both in the height of the individual line shape and in the separations between the side peaks and the central one [cf. Figs. 12(c) and 12(d)]. At the lower laser frequency, e.g., Fig. 12(c), the GVV triple peaks are lower and more distanced than the RWA ones and vice versa at the higher laser frequency, e.g., Fig. 12(d). This can be attributed to the fact that the GVV resonance spectrum $\bar{\rho}_{b b}$ is shifted to the right away from the RWA counterpart [cf. Fig. 12(a)]: At the lowerfrequency side, e.g., $\omega_{L}=90.0$, the GVV calculations predict a smaller long time-averaged population $\bar{\rho}_{b b}$ of the upper level $b$ and vice versa. The difference, both in $\bar{\rho}_{b b}$ and $\bar{I}(\omega)$, between the GVV and the RWA results at the one-photon resonance is expected to gradually diminish as we further reduce the magnitude of the Rabi frequency $\left|V_{a b}\right|$ and as the Bloch-Siegert shift becomes increasingly negligible. 

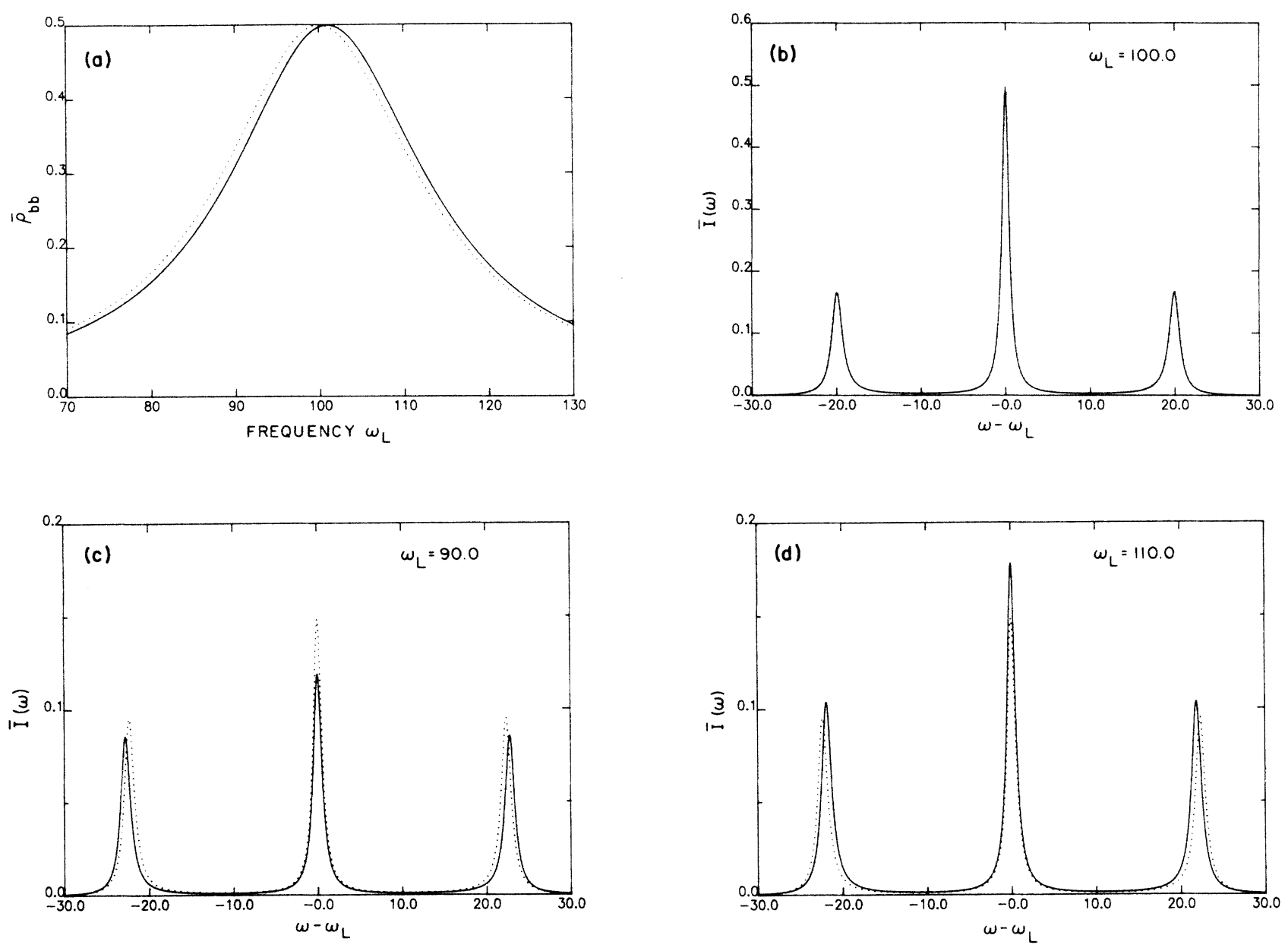

FIG. 12. (a) Long time-averaged population $\bar{\rho}_{b b}$, as a function of $\omega_{L}$, of the level $|b\rangle$ and (b), (c), and (d) fluorescence power spectra $\bar{I}(\omega)$ at $\omega_{L}=100.0,90.0$, and 110.0, respectively. Parameters used are $\omega_{b a}=100.0, \gamma_{b a}=1.0, \gamma_{a b}=0.0,\left|V_{a b}\right|=10.0$, and $\phi^{(1)}=0.0$ (arbitrary units). Solid curves, GVV; dotted curves, RWA.

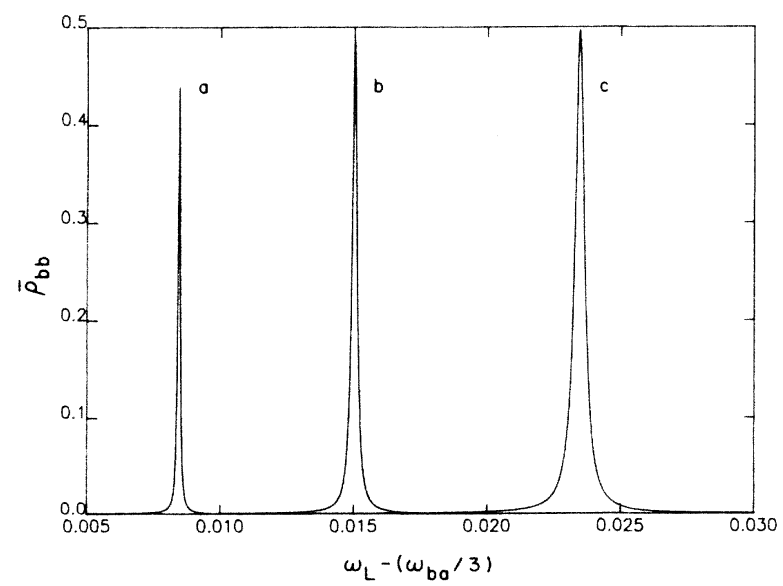

FIG. 13. Long time-averaged populations $\bar{\rho}_{b b}$, as functions of the laser frequency $\omega_{L}$, at $a,\left|V_{a b}\right|=0.75 ; b,\left|V_{a b}\right|=1.0$; and $c,\left|V_{a b}\right|=1.25$; respectively. Parameters used are $\omega_{b a}=100.0$, $\gamma_{b a}=1.0 \times 10^{-4}, \gamma_{a b}=0.0$, and $\phi^{(1)}=0.0$ (arbitrary units). Results are obtained from the GVV calculations.

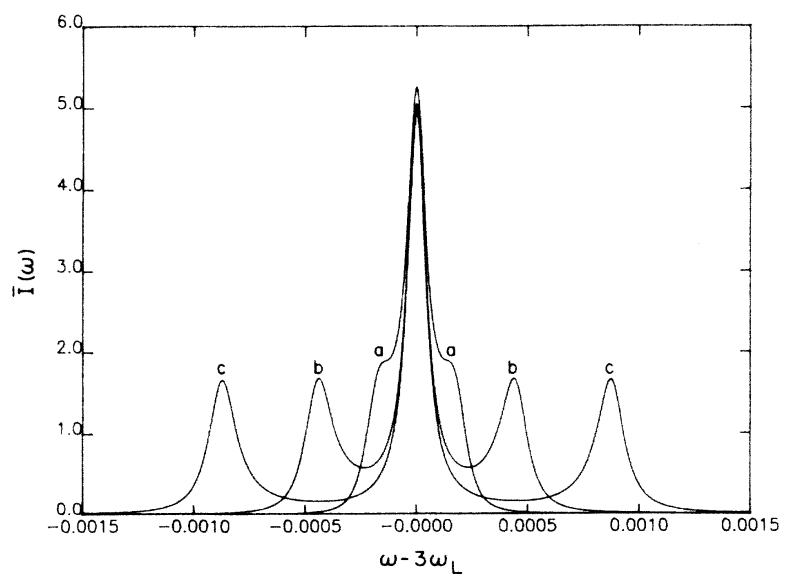

FIG. 14. Three-photon resonance fluorescence power spectra $\bar{I}(\omega) \times 10^{3}$ at the shifted resonance laser frequencies $a$, $\omega_{L}=33.341771 ; b, \omega_{L}=33.348335$; and $c, \omega_{L}=33.356775$ corresponding to $\left|V_{a b}\right|=0.75,1.0$, and 1.25 , respectively. Parameters used are $\omega_{b a}=100.0, \gamma_{b a}=1.0 \times 10^{-4}, \gamma_{a b}=0.0$, and $\phi^{(1)}=0.0$ (arbitrary units). Results are obtained from the GVV calculations. 


\section{Three-photon resonance case: $\gamma_{b a}=1.0 \times 10^{-4}$ and $\left|V_{a b}\right|=0.75,1.0$, and 1.25 (arbitrary units)}

As pointed out previously, in the multiphoton resonance case, i.e., $\omega_{b a} \approx(2 n+1) \omega_{L}, n \neq 0$, the resonance occurs at a shifted laser frequency which is orders of magnitude greater than the corresponding width of the resonance spectrum $\bar{\rho}_{b b}\left(\omega_{L}\right)$, unlike in the one-photon case. In Fig. 13 we present the three-photon resonance spectra $\bar{\rho}_{b b}$, as functions of laser frequency $\omega_{L}$, at three different $\left|V_{a b}\right|$, i.e., $0.75,1.0$, and 1.25. We note the following. (i) The magnitude of the resonance shift is much larger than the corresponding width of the resonance line shape; the width increases, of course, as $\left|V_{a b}\right|$ increases. (ii) The height of the resonance peak at smaller $\left|V_{a b}\right|$, e.g., 0.75 , is lower than that at the larger $\left|V_{a b}\right|$, e.g., 1.25, due to the competition between the pumping rate from the lower level $a$ to the upper level $b$ by the laser field and the spontaneous decay rate from the level $b$ to the level $a$. As the Bloch-Siegert shifts are rather large, it is apparent that when the laser frequency $\omega_{L}$ is exactly equal to $\omega_{b a} / 3$, the unshifted three-photon resonance frequency, the resonance transition between the two levels is completely quenched, in contrast to the one-photon resonance case.

The three-photon resonance fluorescence power spectra at $\left|V_{a b}\right|=0.75,1.0$, and 1.25 are shown in Fig. 14 at each individual shifted resonance frequency, namely, $\omega_{L}=33.341771,33.348335$, and 33.356775, respectively. The familiar three-peak line shape seen at the one-photon resonance case is observed again at the shifted threephoton resonance case: the symmetry of the side peaks about the central one and the relative ratio of the heights and the widths of peaks. We should note that (i) at an even higher multiphoton resonance case, as long as the laser frequency $\omega_{L}$ can be exactly tuned to the corresponding shifted resonance frequency, the induced threepeak fluorescence light spectrum can be intense enough and become detectable, and (ii) the three-photon case discussed here has been modeled in such a way that it closely resembles a real two-level system (such as the $\mathrm{Na}$ atom) as far as the relative magnitude of the spontaneous decay rate $\gamma_{b a}$ and the energy difference $\omega_{b a}$ of the two levels is concerned.

\section{CONCLUSIONS}

In summary, we have shown that the many-mode Floquet theory ${ }^{5}$ can be extended to treat the time-dependent Liouville equation, and that the resulting generalization provides a powerful time-independent nonperturbative technique for the treatment of intense field multiphoton processes (undergoing relaxations) in polychromatic fields. In addition, the GVV-Liouvillian approach allows a natural higher-order perturbative extension of the conventional RWA limit, providing useful analytical results and new physical insights regarding various nonlinear optical phenomena. Extension of the method to the study of Raman scattering, collisional redistribution of radiation, renormalized nonlinear susceptibility, etc., is in progress.

\section{ACKNOWLEDGMENTS}

This work was supported in part by the U.S. Department of Energy (Division of Chemical Sciences) and the American Chemical Society-Petroleum Research Fund. S.-I C. acknowledges partial support by the Joint Institute of Laboratory Astrophysics (JILA) Visiting Fellow Program, and stimulating and instructive discussions with $\mathbf{J}$. Cooper on resonance fluorescence spectra.
"Permanent address: Department of Chemistry, University of Kansas, Lawrence, KS 66045, where correspondence should be directed.

${ }^{1}$ Recent reviews can be found, for example, in Coherent Nonlinear Optics, edited by M. S. Feld and V. S. Letokhov (Springer, New York, 1980); P. A. Schulz, Aa. S. Suadbo, D. J. Krajnovich, H. S. Kwok, Y. R. Shen, and Y. T. Lee, Annu. Rev. Phys. Chem. 30, 311 (1979); Advances in Multiphoton Processes and Spectroscopy, edited by S. H. Lin (World Scientific, Singapore, 1984), Vol. 1.

${ }^{2}$ Y. R. Shen, The Principles of Nonlinear Optics (Wiley, New York, 1984); M. D. Levenson, Introduction to Nonlinear Laser Spectroscopy (Academic, New York, 1982).

${ }^{3}$ See, for example, L. Allen and J. H. Eberly, Optical Resonance and Two-Level Atoms (Wiley, New York, 1975); M. Sargent, M. O. Scully, and W. E. Lamb, Jr., Laser Physics (AddisonWesley, Reading, Mass., 1974).

${ }^{4}$ For a more recent review on Floquet approaches, see S.-I Chu, Adv. At. Mol. Phys. 21, 197 (1985), and references therein.

5 (a) T.-S. Ho, S.-I Chu, and J. V. Tietz, Chem. Phys. Lett. 99, 422 (1983); (b) S.-I Chu and T.-S. Ho, Isr. J. Chem. 24, 237 (1984); (c) T.-S. Ho and S.-I Chu, J. Phys. B 17, 2101 (1984); (d) Phys. Rev. A 31, 659 (1985); (e) 32, 377 (1985).

${ }^{6}$ There are several previous attempts at removing the time dependence of the interaction of atoms with fields. See, for example, A. Ben-Reuven and Y. Rabin, Adv. Chem. Phys. 47,
555 (1981), and references therein. However, there are no explicit treatments of multiphoton processes beyond the RWA limit using the supermatrix approach.

${ }^{7}$ R. G. Breene, Jr., Theories of Spectral Line Shape (Wiley, New York, 1981), and references therein.

${ }^{8}$ U. Fano, Phys. Rev. 131, 259 (1963).

${ }^{9}$ B. R. Mollow, Phys. Rev. 188, 1969 (1969).

10(a) B. R. Mollow, Phys. Rev. A 2, 76 (1970); (b) 5, 1522 (1972); (c) 5, 2217 (1972); (d) 12, 1919 (1975).

${ }^{11}$ H. J. Carmichael and D. F. Walls, J. Phys. B 8, L77 (1975); 9, 1199 (1976).

12S. S. Hassan and R. K. Bullough, J. Phys. B 8, L147 (1975).

${ }^{13}$ S. Swain, J. Phys. B 8, L437 (1975); Adv. At. Mol. Phys. 16, 159 (1980).

${ }^{14}$ H. J. Kimble and L. Mandel, Phys. Rev. A 13, 2123 (1976).

${ }^{15}$ C. Cohen-Tannoudji, in Frontiers in Laser Spectroscopy, edited by R. Ballian, S. Haroche, and S. Liberman (Wiley, New York, 1977), p. 103.

${ }^{16}$ F. Shuda, C. R. Stroud, Jr., and M. Hercher, J. Phys. B 7, L198 (1973).

${ }^{17}$ W. Hartig, W. Rasmussen, R. Schieder, and H. Walther, Z. Phys. A 278, 205 (1976).

${ }^{18}$ R. E. Grove, F. Y. Wu, and S. Ezekiel, Phys. Rev. A 15, 227 (1977).

${ }^{19}$ V. P. Krainov and A. V. Kruglikov, Opt. Spektrosk. 43, 103 (1977) [Opt. Spectrosc. (USSR) 43, 56 (1977)]. 
${ }^{20}$ B. Kirtman, J. Chem. Phys. 49, 3890 (1968); 75, 798 (1981).

${ }^{21}$ P. R. Certain and J. O. Hirschfelder, J. Chem. Phys. 52, 5977 (1970); J. O. Hirschfelder, Chem. Phys. Lett. 54, 1 (1978).

22B. Renaud, R. M. Whitley, and C. R. Stroud, Jr., J. Phys. B 10, 19 (1976).

${ }^{23}$ E. Courtens and A. Szöke, Phys. Rev. A 15, 1588 (1977).

24J. H. Eberly and K. Wódkiewicz, J. Opt. Soc. Am. 67, 1252 (1977).

${ }^{25}$ G. Nienhuis, J. Phys. B 16, 2677 (1983).

${ }^{26}$ (a) J. H. Eberly, C. V. Kunasz, and K. Wódkiewicz, J. Phys. B 13, 217 (1980); (b) X. Y. Huang, R. Tanaś, and J. H. Eberly, Phys. Rev. A 26, 892 (1982); (c) P. D. Kleiber, J. Cooper, K. Burnett, C. V. Kunasz, and M. G. Raymer, ibid. 27, 291 (1983).

${ }^{27}$ B. Dick and R. M. Hochstrasser, Chem. Phys. 75, 133 (1983); Chem. Phys. Lett. 102, 484 (1983); J. Chem. Phys. 81, 2897 (1984), and references therein.

${ }^{28}$ The procedure of removing the time dependence of Eq. (9) is different from that introduced in Ref. 6 and others in that we have mathematically proved, in terms of many-mode Floquet theory [see Ref. 5(a)], that the transformation is exact, whereas those authors in Ref. 6 and others simply expand the density-matrix operator in terms of multiple Fourier components.

${ }^{29}$ Also see (a) J. H. Shirley, Phys. Rev. 138, B979 (1965); (b) D. R. Dion and J. O. Hirschfelder, Adv. Chem. Phys. 35, 265 (1976); (c) K. Aravind and J. O. Hirschfelder, J. Phys. Chem. 88, 4788 (1984) where a two-level system in a single monochromatic intense laser field has been studied in great detail.

${ }^{30}$ The procedure of Laplace transform has been employed widely (see, e.g., Refs. 9 and 10), but in our exact treatment in Sec. II, matrix manipulation is more expedient.

${ }^{31}$ R. Loudon, The Quantum Theory of Light, 2nd ed. (Oxford University Press, New York, 1983).

${ }^{32}$ M. Lax, Phys. Rev. 129, 2342 (1963); 172, 350 (1968). 